\title{
Resting-state and vocabulary tasks distinctively inform on age-related differences in the functional brain connectome
}

\author{
Perrine Ferré ${ }^{a}$, Yassine Benhajali ${ }^{a}$, Jason Steffener ${ }^{a, b, c}$, Yaakov Stern $^{d}$, Yves Joanette ${ }^{a}$ and Pierre Bellec ${ }^{a}$ \\ ${ }^{a}$ Centre de recherche de I'Institut Universitaire de Gériatrie de Montréal (CRIUGM), Université de Montréal, Montreal, Quebec, Canada; \\ bPERFORM Center, Concordia University, Montreal, Quebec, Canada; ' Interdisciplinary School of Health Sciences, University of Ottawa, Ottawa, \\ Ontario, Canada; ${ }^{\mathrm{d}}$ Cognitive Neuroscience Division, Columbia University, New York, NY, USA
}

\section{ABSTRACT}

Most of the current knowledge about age-related differences in brain neurofunctional organisation stems from neuroimaging studies using either a "resting state" paradigm, or cognitive tasks for which performance decreases with age. However, it remains to be known if comparable agerelated differences are found when participants engage in cognitive activities for which performance is maintained with age, such as vocabulary knowledge tasks. A functional connectivity analysis was performed on 286 adults ranging from 18 to 80 years old, based on resting state or vocabulary tasks. Increases in connectivity of regions of the language network were observed during task completion. Conversely, only age-related decreases were observed during resting-state. While vocabulary accuracy increased with age, no interaction was found between functional connectivity, age and task accuracy or proxies of cognitive reserve, suggesting that older individuals typically benefits from semantic knowledge accumulated throughout one's life trajectory, without the need for compensatory mechanisms.

\section{ARTICLE HISTORY}

Received 22 May 2018

Accepted 5 March 2019

\section{KEYWORDS}

Aging; functional

connectivity; vocabulary;

resting state

\section{Introduction}

By 2050, the number of individuals over the age of 60 is projected to have more than tripled by reference to 2000, (United Nations, 2017). As a consequence, the incidence of cognitive impairments is set to increase sharply. Cognitive impairments are the most feared health conditions associated with aging amongst younger adults (Anderson, Day, Beard, Reed, \& Wu, 2009; CDC, 2013). Cognitive health is central to quality of life (WHO | What is Healthy Ageing?, 2018). Language abilities in particular allow for the creation or maintenance of inter-personal relationships, the basis of social participation.

Cognitive abilities in general depend on a large and dynamic brain architecture that is known to change as we age (Andrews-Hanna et al., 2007; Damoiseaux et al., 2008; Marstaller, Williams, Rich, Savage, \& Burianová, 2015; Sporns, 2014). It is thus important to go beyond studies of isolated regions and explore the capacity of different brain regions to interact together.

Functional connectivity (FC) has proven to be a reliable tool for capturing statistical dependencies between the activities of distant regions. FC measures have repeatedly been shown to be associated with age (e.g. Cao et al., 2014; Chételat et al., 2013; Damoiseaux et al., 2008; Li et al., 2012; Perry et al., 2015). The vast majority of FC studies in aging has relied on a resting state (RS) paradigm, rather than a task, and has focused on a small number of functionally meaningful regions, such as the default mode network (DMN). Brain areas of the DMN are active at rest or when individuals are engaged in internal thoughts (e.g. autobiographical memory retrieval, envisioning the future), but critically deactivate during externally oriented tasks (Buckner, Andrews-Hanna, \& Schacter, 2008). The RS paradigm has gained popularity and is now considered a gold standard for the study of brain organisation and cognitive mechanisms in aging. For some authors, an RS paradigm would reveal a somewhat "universal" functional architecture (Ito et al., 2017; Krienen, Yeo, \& Buckner, 2014). The structure of the networks observed at rest is indeed claimed to share up to $80 \%$ of the variance with that observed during cognitive tasks (Cole, Bassett, Power, Braver, \& Petersen, 2014) and show significant association with structural connectivity (Hagmann et al., 2008). RS is also thought to overcome potential confounds associated with task-based paradigm, such as a lower signal to noise ratio and artificial co-fluctuations driven by the temporal structure of the task (Birn, Diamond, Smith, \& Bandettini, 2006; Hillenbrand, Ivry, \& Schlerf, 2016; Sadaghiani \& Kleinschmidt, 
2013). As such, RS-FC is being advocated by some as a potential "functional fingerprint" (lordan \& ReuterLorenz, 2016), a trait, that is highly predictive of taskrelated activation (Chan, Alhazmi, Park, Savalia, \& Wig, 2017) and cognitive behaviour (Damoiseaux et al., 2008).

But a number of authors have raised concerns (Campbell \& Schacter, 2017; Davis, Stanley, Moscovitch, \& Cabeza, 2017; Geerligs \& Tsvetanov, 2017; Grady, 2016) about the small number of FC studies that use taskbased designs or report language regions (see Campbell and Schacter's review article (2017)), as well as the limitations of an RS paradigm when addressing questions specific to cognition. For example, the actual cognitive state of the participant may be difficult to monitor during an RS acquisition (Davis et al., 2017) and many note a tendency to fall asleep, which can dramatically affect functional connectivity patterns (Haimovici, Tagliazucchi, Balenzuela, \& Laufs, 2017; Wang, Han, Nguyen, Guo, \& Guo, 2017).

Also, semantic networks identified using RS are not identical to those obtained from task-based FC (Jackson, Hoffman, Pobric, \& Lambon Ralph, 2015). Critical to the present exploration, age-related differences in RS connectivity appear to generalise poorly across different cognitive tasks (Grady, 2016; Mennes, Kelly, Colcombe, Castellanos, \& Milham, 2013; Spreng \& Schacter, 2012). Evidence rather points towards domain-specific strategic processes and differential involvement of functional brain networks in aging (Geerligs, Rubinov, CamCAN, \& Henson, 2015; Meunier, Stamatakis, \& Tyler, 2014; Samu et al., 2017).

Considering that age does not affect all cognitive abilities at the same level, there exist a strong motivation to study aging of brain connectivity in other contexts than RS. Intriguingly, in the language domain, some processes appear to be remarkably well preserved. For example, semantic processes, at the heart of knowledge representation, appear relatively well maintained during the aging trajectory. A classic conceptualisation of vocabulary knowledge includes a combination of semantic, phonological or orthographic information retrieved either to understand or to produce words. Different test formats are available to examine knowledge representation (e.g. semantic judgment, picture naming, word definition). Together, these tests provide a way to examine vocabulary constructs through different access routes to semantic information (Salthouse, 2014).

The ability to understand the meaning of written or oral words (e.g. semantic judgment, word association) is typically spared until 65 years of age (Salthouse, 2014) and beyond (Fabiani, 2012; Schaie \& Willis, 2010; Verhaeghen, 2003; Verhaegen \& Poncelet, 2013). Tasks that tap into lexical production (e.g. picture naming, fluency, word definition) bring more inconsistencies, according to the chosen method (see for example Goulet, Ska, \& Kahn, 1994; Shafto, James, Abrams, \& Tyler, 2016). Performance is often similar to that of younger adults, (Shafto et al., 2016) and sometimes better (Salthouse, 2014), especially when accuracy measures, rather than response times, are used (Verhaegen \& Poncelet, 2013) in a constrained (Schmitter-Edgecombe, Vesneski, \& Jones, 2000) and untimed task (Wieranga et al., 2008). In effect, both active and passive vocabulary thus appear to be behaviourally preserved when task demand is controlled, at least until 65 years of age (Salthouse, 2014).

The majority of task-based FC investigation of cognitive aging tapped into abilities known to weaken with age (for a review see Sala-Llonch, Bartrés-Faz, \& Junqué, 2015). Evidence thus remains scarce and inconsistent with respect to the functional brain mechanisms underpinning the maintenance of semantic knowledge with age. Among the few studies that have used word production or comprehension tasks, some report an agerelated cerebral dedifferentiation, i.e. a reduction in hemispheric asymmetry (Chan et al., 2017; La et al., 2016) or increases in connectivity between regions of the language network (Hoyau et al., 2018). Such patterns are atypical for younger adults and suggestive of compensatory mechanisms. In addition, the interplay of the DMN with language and domain-general networks seems crucial to explain cognitive performance of older adults (Dong et al., 2012; Meinzer et al., 2012; Muller, Mérillat, \& Jäncke, 2016; Muller \& Meyer, 2014).

In addition to the effect of age or performance, FC could further be modulated by individual factors acquired through life experiences, such as cognitive reserve. Cognitive reserve is a hypothetical construct, hypothesised to promote efficiency, maintenance or compensation of neural circuitry and consequently contribute to preserved cognition in aging (Stern, 2009). Cognitive reserve is estimated indirectly, using measures such as education level, vocabulary skills and intelligence. These variables presumably reflect life experiences, above and beyond that of age, and have been used in the literature as proxies for cognitive reserve, either in isolation or in combination. Previous explorations suggest that cognitive reserve is associated with greater functional connectivity during RS (Bozzali et al., 2015; Franzmeier, Hartmann, et al., 2017; Marques et al., 2016) and tasks (Guzzetti \& Daini, 2014), and supports better cognitive performance in the face of aging (Bozzali et al., 2015; Franzmeier, Buerger, et al., 2017; Tucker-Drob \& Salthouse, 2011). Here again, investigations were conducted either with an RS paradigm or with cognitive tasks characterised by a progressive 
diminution of performance with age. To our knowledge, cognitive reserve estimates have never been investigated with FC in relation to age during tasks which involve maintained abilities, such as vocabulary.

Also, despite the presence of competing points of view in the literature between RS and task-based paradigms, no study directly compares age-related differences in connectivity during vocabulary tasks with those observed in RS. It remains to be known whether age-related differences in FC will vary according to the cognitive context, and inside the same construct across different vocabulary tasks in particular.

Given that the neurofunctional basis of cognitive aging have mostly been explored with RS paradigm or tasks which involve a decrease in performance, a connectome-wide exploration of age effect on FC associated with different cognitive states will contribute to disentangle the mechanisms underlying the behavioural preservation of vocabulary knowledge in healthy aging.

The general goal of this study is thus to describe and compare the age-related differences in FC associated with RS and vocabulary tasks, with a focus on DMN and language regions' connectivity. More specifically, the first main objective is to assess the association between age and accuracy for vocabulary tasks as well as tasks from other cognitive domains. The second objective is to evaluate the association between age and functional connectivity for each cognitive state as well as the main association or interaction with variables such as cognitive reserve estimates or task accuracy. The third objective is to quantify similarities in age effect between and within cognitive states. It was first anticipated that vocabulary accuracy would generally be maintained with age, in dissociation with other cognitive tasks. Second, functional connectivity of the whole connectome is expected to mainly decrease with age, in particular in respect to the DMN activity, with some increases in the connectivity of language regions during task performance. Accuracy measures as well as cognitive reserve estimates are hypothesised to be associated with FC for the whole sample, but also to influence the effect of age on functional connectivity (e.g. an interaction between the effect of age and cognitive reserve on $\mathrm{FC}$ increases) during vocabulary tasks in particular. Third, the effect of age on FC is expected to vary according to the induced cognitive state, regardless of the experimental paradigm, noise or potential confound parameters.

\section{Methods}

To answer our three main objectives, a cohort of 300 participants were scanned with $\mathrm{fMRI}$ during a RS paradigm and three vocabulary tasks. We conducted a series of experiments which have been summarised in Table 1.

\subsection{Participants}

Data analysed in this paper are a subset of those collected as part of the Reference Ability Neural Network (RANN) study (Stern et al., 2014). The overall objective of the RANN study is to "determine whether spatial fMRI networks that are uniquely associated with the performance of each reference ability can be derived." (Stern et al., 2014). The present project focuses on the language behavioural and imaging (MRI) domains, with the addition of RS. Among the 301 participants that were recruited, 286 individuals qualified after $\mathrm{fMRI}$ quality control and behavioural screening, with ages ranging from 20 to 80 years (Mean age $=48.45, S D=17.13$ ) (see Table 2 for distributions of sex and education across age continuum). All participants gave informed consent according to Columbia University Medical Center Ethical Committee (CF-AAAS8207), USA, between December 2015 and June 2016. Secondary analysis of the RANN sample for the purposes of this study was approved by the local ethics committee at the "Centre de Recherche de I'Institut Universitaire de Gériatrie de Montréal", University of Montréal, Canada. Participants were native English speakers, right-handed, and free of medical or psychiatric conditions that could affect cognition. All individuals underwent extensive screening questionnaires to exclude participants with MRI contraindications, hearing, visual perception and functional impairment, with a Mattis Dementia Rating Scale greater than 135 (Mattis, 1988).

\subsection{Tasks}

Previous studies have mostly examined vocabulary knowledge through a single measure. Different formats of vocabulary tests provide insights into different access routes to semantic information (Salthouse, 2014). Three tasks were thus selected to reflect word

Table 1. Participants' demographics and cognitive testing.

\begin{tabular}{|c|c|c|c|}
\hline Age range $(N)$ & $20-35(93)$ & $36-59$ (106) & $60-80(87)$ \\
\hline $\begin{array}{l}\text { Mean years of } \\
\text { education } \\
\text { [min-max] }\end{array}$ & 16 [9-24] & 16 [11-22] & 17 [12-22] \\
\hline $\begin{array}{l}\text { Sex (male/female) } \\
\text { Mean accuracy } \\
(s d)[\text { min-max] }\end{array}$ & $60 / 33$ & $52 / 54$ & $40 / 47$ \\
\hline Antonyms & $50(1$ & 53( & $60(2)-2)$ \\
\hline Synonyms & $53(21)[7-86]$ & 59 (22) [20-100] & 69 (21) [27-100] \\
\hline Picture Naming & 41 (17) [10-80] & $48(20)[8-90]$ & 58 (16) [21-86] \\
\hline WAIS-Matrix & $42(10)[9-56]$ & 37 (13) [9-57] & $34(13)[9-100]$ \\
\hline & $42(10)[9-56]$ & 37 (13) [9-57] & 34 (13) [9-100] \\
\hline WAIS-Letter Numbe & $68(16)[30-10$ & 58 (13) [32-94] & 48 (11) [22-78] \\
\hline
\end{tabular}


Table 2. Summary of the specific objectives, experiments and findings of the study.

\begin{tabular}{|c|c|c|}
\hline Specific objectives and hypotheses & Experiments & Main findings \\
\hline $\begin{array}{l}\text { 1. Assess the association between age and } \\
\text { accuracy for vocabulary tasks as well as other } \\
\text { cognitive tests. } \\
\text { A positive association is expected between age and } \\
\text { vocabulary accuracy, and a negative association } \\
\text { for other behavioural domains. }\end{array}$ & $\begin{array}{l}\text { Pearson correlation coefficients were computed } \\
\text { with age and task accuracy as continuous } \\
\text { variables. }\end{array}$ & $\begin{array}{l}\text { There was a significant correlation between age and } \\
\text { accuracy across all tasks. Increasing age was } \\
\text { positively associated with accuracy for vocabulary } \\
\text { tasks, whereas the association was negative for } \\
\text { other cognitive domains (Figure } 2 \text { ). }\end{array}$ \\
\hline $\begin{array}{l}\text { 2.a. Explore average connectivity at the sample } \\
\text { level and evaluate associations with task } \\
\text { accuracy or cognitive reserve estimates } \\
\text { during tasks and rest. } \\
\text { Significant associations were expected in the } \\
\text { connectome for all tasks, as well as rest, regardless } \\
\text { of age. In particular, association with cognitive } \\
\text { reserve estimates as well as vocabulary accuracy } \\
\text { were expected in brain connectivity of both } \\
\text { language and default mode network regions. }\end{array}$ & $\begin{array}{l}\text { A connectome-wide association analysis was } \\
\text { computed for each cognitive state. A general } \\
\text { linear model (GLM), controlling for age, tested } \\
\text { the association between each variable and } \\
\text { functional connectivity, for all pairs within the } \\
53 \text { parcels. For the four selected regions of } \\
\text { interest, Cohen's } f^{2} \text { (Cohen, 1988) was used to } \\
\text { visualise spatial maps of the effect size. }\end{array}$ & $\begin{array}{l}\text { Average patterns of connectivity are coherent with } \\
\text { previously reported DMN and language networks } \\
\text { (Figure 3). Associations between accuracy or } \\
\text { cognitive reserve estimates on brain connectivity } \\
\text { were heterogeneously found, with no consistent } \\
\text { pattern. }\end{array}$ \\
\hline $\begin{array}{l}\text { 2.b. Evaluate the association between age and } \\
\text { functional connectivity for each cognitive } \\
\text { state. } \\
\text { An association in connectivity and age was } \\
\text { expected across all cognitive states. Mostly } \\
\text { decreases in connectivity were previously reported, } \\
\text { but increases may also be observed. Such increase } \\
\text { would in particular be observed during tasks in- } \\
\text { between the selected language regions and with } \\
\text { domain-general regions. }\end{array}$ & $\begin{array}{l}\text { Same as } 2 \mathrm{a} \text {, with different predictive variables and } \\
\text { controlling for sex. }\end{array}$ & $\begin{array}{l}\text { Significant association between age and connectivity } \\
\text { were found throughout the connectome. } \\
\text { Significant decreases as well as increases were } \\
\text { found in particular in all of the four selected parcels } \\
\text { (Figure 4). }\end{array}$ \\
\hline $\begin{array}{l}\text { 2.c. Test the interaction between age and task } \\
\text { accuracy or cognitive reserve estimates on } \\
\text { functional connectivity. } \\
\text { Significant interaction effects were expected. In } \\
\text { particular, an interaction was expected between } \\
\text { age and accuracy, the cognitive reserve index and } \\
\text { education. }\end{array}$ & $\begin{array}{l}\text { Same as } 3 a \text {, adding the second variable of interest } \\
\text { in the model }\end{array}$ & $\begin{array}{l}\text { Only the interaction between age and years of } \\
\text { education - exclusively during resting-state - } \\
\text { brought substantial and significant connectome- } \\
\text { wide changes. }\end{array}$ \\
\hline $\begin{array}{l}\text { 2.d. Assess the impact of potential confounds in } \\
\text { the choice of regression parameters (sex, } \\
\text { movement, brain atrophy) on the age-FC } \\
\text { associations reported in } \mathbf{2 b} \text {. } \\
\text { The associations were expected to be robust to the } \\
\text { inclusion of confounding variables. }\end{array}$ & $\begin{array}{l}\text { In further models, the regression analysis was } \\
\text { replicated by cumulatively adding confounding } \\
\text { variables. }\end{array}$ & $\begin{array}{l}\text { The various models showed overall very high } \\
\text { agreement (Figure } 8 \text { ). Only sex was thus included } \\
\text { as a confound variable in our GLM regression } \\
\text { model. }\end{array}$ \\
\hline $\begin{array}{l}\text { 3.a. Quantify similarities in age effect between } \\
\text { cognitive states. } \\
\text { The effect of age on functional connectivity was } \\
\text { expected to be dependent on the cognitive state, } \\
\text { with a higher similarity between word } \\
\text { comprehension and production tasks than between } \\
\text { tasks and rest. }\end{array}$ & $\begin{array}{l}\text { A Pearson's spatial correlation coefficient was } \\
\text { used to quantify the similarity between age }\left(f^{2}\right) \\
\text { effect maps across the different cognitive states } \\
\text { for each ROI (Figure 5). }\end{array}$ & $\begin{array}{l}\text { Age effect maps had the highest spatial similarity } \\
\text { during antonyms and synonyms tasks. Age effect } \\
\text { maps during picture naming was less similar with } \\
\text { both antonyms/synonyms and rest. Resting state } \\
\text { was most dissimilar (Figure 5). }\end{array}$ \\
\hline $\begin{array}{l}\text { 3.b. Validate the reliability of age effect within } \\
\text { cognitive states. } \\
\text { Age effect on functional connectivity was expected } \\
\text { to be relatively consistent within each cognitive } \\
\text { state, indicating that age-effects are mostly driven } \\
\text { by the cognitive state rather than random noise or } \\
\text { the design of the experimental paradigm. }\end{array}$ & $\begin{array}{l}\text { Analyses were replicated using a split-half } \\
\text { procedure and by extracting only data points } \\
\text { within task blocks instead of the full run. }\end{array}$ & $\begin{array}{l}\text { A strong spatial correlation between age maps was } \\
\text { observed when comparing split-half, intra-blocks } \\
\text { and whole run models within each cognitive state } \\
\text { (Figure 6). In comparison, age effects are much } \\
\text { more variable when comparing one cognitive state } \\
\text { to another. }\end{array}$ \\
\hline
\end{tabular}

semantic knowledge. Participants completed two word comprehension tasks (synonyms and antonyms judgment) and one expressive lexical retrieval task (picture naming). The synonyms and antonyms tasks required subjects to match a written probe word to its synonym or antonym, respectively (Salthouse \& Kersten, 1993). The probe word was presented on the screen along with four written choices for either antonym or synonym, and participants indicated their choice using a button press. The task began with a 36-second fixation cross, had five 42-second task blocks interspersed with 28-second fixation blocks, for a total of 5 blocks of three items each. Each item was presented for $13.5 \mathrm{~s}$ with a $500 \mathrm{~ms}$ inter-stimulus interval between items. Accuracy score was defined as the proportion of synonyms or antonyms correctly identified in the time constraint. Response times were also computed for the synonyms and antonyms tasks. The picture naming task presented 40 coloured bitmap images to the participants, who had to name these images out loud, adapted from the picture naming task of the WJ-R Psycho-Educational battery (Woodcock, Johnson, \& Mather, 1989). The task began with a 36-second fixation-cross and there was a 28-second fixation time between blocks, 
for a total of five 40-second blocks. Each picture was presented for $4.5 \mathrm{~s}$ with a $500-\mathrm{ms}$ ISI between items. Accuracy (not response time) was computed online, as defined by the proportion of pictures correctly named in the time constraint. Fixed criteria (Salthouse, 1998) were used to define a correct answer, with two exceptions. First, credit was given if the correct word was included in the subject's response (e.g. "printing press" for "press"). Second, a slight mispronunciation of the correct word also received credit (e.g. "cumberbund" for cummerbund). General cognitive measures were also collected in the scanner with computerised subtests adapted from the Wechsler Adult Intelligence Scale (WAIS-3) (Wechsler, 1997). Focus was given on the accuracy score (proportion of correct responses) for the Matrix, Digit-Symbol and Letter-Number subtests, measures of fluid reasoning, perceptual speed and working memory, respectively. The Vocabulary subtests of the WAIS-3 were also used as a proxy of cognitive reserve. Incomplete tasks (due to technical issues or unfinished assessments) were discarded, as well as extreme values (defined by $1.5 \mathrm{x}$ interquartile range, SPSS).

\subsection{Imaging protocol}

All magnetic resonance images were acquired on a 3.0 T Philips Achieva Magnet. Each session started with a scout T1-weighted image, to determine patient position and adjust the $240 \mathrm{~mm}$ field of view. In total, there were two, 2-hour MR imaging sessions to accommodate the functional acquisitions of the twelve tasks as well as all other imaging runs. The presentation order of the tasks did not vary within session, but the order of the two sessions was counterbalanced across subjects. Three functional imaging runs collected vocabulary tasks ( 6 mins and $30 \mathrm{~s}$ ) following a block design. An additional resting BOLD functional run $(7 \mathrm{~min})$ was acquired. All fMRI acquisitions used an EPI sequence with 41 axial slices; TE/TR (ms) 20/2000; Flip Angle (deg) $72^{\circ}$; inplane resolution (voxels) $112 \times 112$; slice thickness/gap (mm) 3/0. In our sample of 286 participants, 128 individuals had their $\mathrm{T} 1$ done in a separate session. Seven individuals had their RS acquired at a different session than their language tasks. A neuroradiologist reviewed each subject's scans for incidental finding, which were conveyed to the subject's primary care physician.

\subsection{Image preprocessing}

The $\mathrm{fMRI}$ data were preprocessed using the pipeline implemented in the neuroimaging analysis kit (NIAK, http://niak.simexp-lab.org/pipe_preprocessing.html), including the following steps.

Registration. The three first volumes of each run were removed to allow the magnetisation to reach equilibrium. Individual time series were corrected for interslice difference in acquisition time and rigid body motion (within and between runs). For each subject, the median volume of one arbitrary reference run was co-registered with a T1 individual scan using Minctracc (Collins, Neelin, Peters, \& Evans, 1994), which was itself nonlinearly transformed to the Montreal Neurological Institute (MNI) nonlinear template (Fonov et al., 2011) using the CIVET pipeline (Ad-Dab'bagh et al., 2006). The rigid-body transform, fMRI-to-T1 transform and T1-tostereotaxic transform were all combined, and the functional volumes were resampled in the MNI space at a $3 \mathrm{~mm}$ isotropic resolution.

Quality control - registration. Each scan for each run was individually reviewed to assess if anatomical/functional alignments were adequate. For cases that failed the quality control, an attempt was made to manually centre the anatomical and functional images prior to automated registration (four individuals). If the registration remained unsatisfactory, the scans were excluded from further analysis (two picture naming runs).

Quality control - motion. It has been shown that participant motion can have large effects on functional connectivity estimates, and motion is more prevalent in older adults, compared to young adults (Mowinckel, Espeseth, \& Westlye, 2012). To minimise such effects, regression of the motion parameters as well as a "scrubbing" procedure was then applied (Power et al., 2014; Power, Barnes, Snyder, Schlaggar, \& Petersen, 2012). This approach decreases spurious correlations related to motion (Yan et al., 2013) and improves network identifiability (Ciric et al., 2017). Scans in which the displacement was larger than $0.5 \mathrm{~mm}$ with respect to the previous scan were removed, as well as the volume before and three volumes after. For each task, time series with less than 60 frames after scrubbing were excluded from further analysis. These criteria led to the exclusion of 42 (out of 299) individual fMRI runs available for the antonyms task, 34 (out of 298) for the synonyms task, and 24 (out of 287) for the RS. The picture naming task generated a lot more head movement in comparison with the other tasks, because participants were asked to verbally name the pictures, which led to the exclusion of 169 functional runs (out of 295).

Noise regression and smoothing. To reduce spurious correlations arising from various noise sources (Giove, Gili, lacovella, Macaluso, \& Maraviglia, 2009), the following nuisance parameters were regressed out from the time series at each voxel: slow time drifts (basis of 
discrete cosines with a $0.01 \mathrm{~Hz}$ high-pass cut-off), average signals in conservative masks of the white matter and the lateral ventricles, as well as the first principal components ( $95 \%$ energy) of the six rigid-body motion parameters and their squares (Lund, Madsen, Sidaros, Luo, \& Nichols, 2006). The fMRI volumes were finally spatially smoothed with a $6 \mathrm{~mm}$ isotropic Gaussian blurring kernel. Global signal regression was not used in the present study, as it is suspected to lead to difficulties in reliably interpreting patterns of negative and positive correlations (Murphy, Birn, Handwerker, Jones, \& Bandettini, 2009; Saad et al., 2012).

\subsection{Brain parcellation and selection of regions of interest}

The present functional connectivity analysis follows a connectome-wide, seed-based approach, using a whole-brain cluster parcellation derived from the sample under study. Functional connectivity was first analysed for all pairs of seeds/parcels, and then four regions of the language and default mode networks were selected for further analysis.

\subsubsection{Data-driven functional parcellation}

Brain parcellation plays a key role in connectivity studies. It is traditionally defined by classic networks observed at rest in a sample of young and healthy participants, but our study specifically aims at comparing cognitive states throughout the age trajectory. Parcellation should thus be unbiased towards a specific cognitive state (task or rest) or age group (younger or older).

Also, the connectivity of language regions is suspected to be unveiled only by task states (Binder, Desai, Graves, \& Conant, 2009; Lohmann et al., 2010; Tomasi \& Volkow, 2012a; Turken \& Dronkers, 2011; Xiang, Fonteijn, Norris, \& Hagoort, 2010): RS fMRI analyses typically aggregate left perisylvian language regions with an extended network of multimodal regions, including the left inferior frontal, posterior temporal and inferior parietal cortices. Parcels were thus generated using an integrative functional parcellation approach (Geerligs, Renken, Saliasi, Maurits, \& Lorist, 2015; Lancichinetti \& Fortunato, 2012), pooling data from all participants, tasks and rest.

A region-growing algorithm was first applied to reduce each $\mathrm{fMRI}$ dataset into a time $x$ space array, with regions of approximately $1000 \mathrm{~mm}^{3}$ each (Bellec et al., 2006). A cluster analysis is then applied on the average time series within each region for all subjects, to identify parcels that consistently exhibit a similar response at the individual level and that are stable at the group level (BASC, Bellec, Rosa-Neto, Lyttelton,
Benali, \& Evans, 2010). Reducing the number of seeds is a straightforward way to mitigate the impact of multiple comparisons (Bellec et al., 2015). The BASC method has been shown to generate group functional parcellations with homogeneity concurrent to state-ofthe-art method (Urchs et al., 2017) and good reproducibility (Garcia-Garcia et al., 2018). As suggested by Sporns, methods such as the BASC overcome the "difficulty to choose a single ('best') representative partition [...] by expressing the optimal partition not as a single 'best' partition but as a meaningful average across multiple near-optimal partitions" (Sporns \& Betzel, 2016). Such multidimensional parcellations derived from functional data have recently shown to outperform anatomical atlases (Sala-Llonch, Smith, Woolrich, \& Duff, 2018).

A multiscale stepwise selection (MSTEPS) method (Bellec, 2013) as well as previous guidelines on statistical power (Bellec et al., 2015) were used to select the more accurate summary of the stable cluster solutions, in the range 10-500. A trade-off between sensitivity and anatomical resolution led us to select 53 clusters as the lowest number of clusters that clearly captured traditional (functional as well as structural) word comprehension and production regions. While connectivity changes were computed at the whole connectome level and were individually checked (i.e. the connectivity of all 53*53 brain parcels), the present study focuses on resting-state and vocabulary tasks. Regions of interest (ROI) were consequently selected a-posteriori among the 53 parcels, in a hypothesis-driven fashion and anchored in previous connectivity studies.

\subsubsection{Regions of interest selection}

With the objective to investigate canonical regions of the language and default mode functional networks, we selected parcels that best overlapped with language and DMN regions reported in previous studies. Four regions of interest among our 53 parcels were thus selected a posteriori (Figure 1).

The DMN was seeded in the posterior cingulate cortex (PCC, mni coordinates [-13 -50 33]). Indeed, both RS fMRI and PET studies show consistently across research sites that brain activity is centralised in posterior regions of the precuneus - PCC, suggesting that it is a central hub for intrinsic brain activity (Andrews-Hanna et al., 2007; Greicius, Krasnow, Reiss, \& Menon, 2003; Kong et al., 2018; Tomasi \& Volkow, 2010).

While vocabulary knowledge likely engage all of the brain to some extent, some regions respond more specifically and strongly when performing word comprehension and production tasks: the posterior superior temporal gyrus (pSTG, including Heschl gyrus (primary 


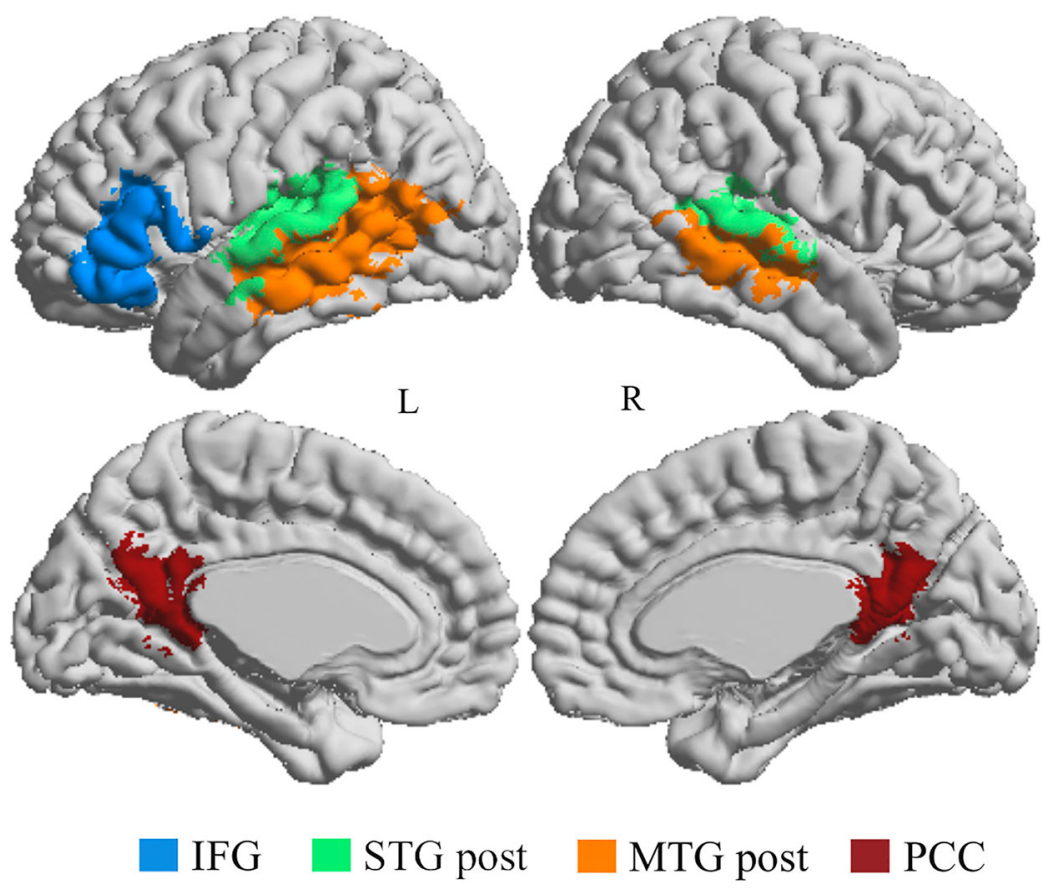

Figure 1. Regions of interest after pooling connectivity data from all cognitive states and from all participants, as defined by a consensus and multilevel cluster analysis (BASC-MSTEPS): PCC: posterior cingulate cortex; IFG: inferior frontal gyrus; STG post: posterior superior temporal gyrus; MTG post: posterior middle temporal gyrus.

auditory area) [-40 -24 7] and planum temporal (Wernicke's region) $[-57-201])$, the inferior frontal gyrus (IFG, including Pars opercularis (Broca region) [-48 13 17] and Pars triangularis (Broca's region) [-47 27 6]), the posterior middle temporal gyrus (pMTG, including the middle temporal gyrus [-59-29-6] and a portion of occipito-temporal area [-47 -52 -12]) (Lohmann et al., 2010; Tomasi \& Volkow, 2012b; Xiang, Dediu, Roberts, Norris, \& Hagoort, 2012).

Although this choice is based on human judgment and visual inspection, one advantage of the clustering approach (as well as ICA) is that "human judgment is incorporated at a high perceptional cluster- or network-level, rather than during the early-stage ROI selection, as is required in traditional seed-based approaches." (Margulies et al., 2010).

\subsection{Statistical analysis}

Behavioural analysis. Vocabulary and general cognitive tests' accuracy were explored in correlation with age. Pearson's bivariate correlation coefficients were used to assess the relationship between age and accuracy scores. Preliminary descriptive analysis was performed to ensure no violation of normality and linearity. Partial correlation was used to explore the relationship while controlling for sex, and education level (in years of schooling), known to significantly associate with cognitive performance.
Functional connectivity measures. Analysis were performed at a connectome-wide level: for each task and each pair of parcel at resolution 53, between-parcels connectivity was measured using the Fisher transform of the Pearson correlation between the average time series of the parcels. The intra-parcel connectivity was the Fisher transform of the average over the correlations between all voxel time series within a parcel. An individual connectome was thus a $53 \times 53$ matrix, with intra-parcel connectivity as diagonal elements, and inter-parcel connectivity off-diagonal.

General Linear Model. A general linear model (GLM) was used to assess the mean sample effect for the whole sample and test the association between phenotypes of interest (e.g. age, education, performance etc.) and the connectome at the group level, for each pair of two regions across all 53 brain regions. While GLM exploration was held at the whole connectome level, further analysis focused on the four selected ROls. Thus, for a given region of interest, the correlation of its signal with the other 52 regions was calculated for each cognitive state. The GLM systematically included an intercept, the age of participants, and sex as co-variables of no interest. The parameters of the model were estimated through minimum least-square, and a t-test with associated $p$-value was derived under a Gaussian, independent and identically distributed assumption on the residuals. A Benjamini-Hochberg false discovery rate (FDR) was implemented to correct for multiple 
comparisons across all brain connections across all 53 regions (Benjamini \& Yekutieli, 2001), with a significance threshold of 0.05 . The validity of the $p$-values was recently investigated on both simulations and a number of real datasets, including "negative control experiments" consisting of comparison of random groups with similar demographic characteristics (Bellec et al., 2015). These evaluations concluded to a tight control of false positive rate to the nominal value.

Statistical contrasts. GLM analyses were used to detect effects for each task separately. Main sample effect was computed preliminarily to obtain an average map of functional connectivity during each cognitive state. The main contrast of interest for this study was age (in years). All covariates except the intercept were corrected to a zero mean. Secondary models tested for associations between connectivity and other individual measures such as task accuracy (respectively antonyms, synonyms, picture naming or the average accuracy score of the three language tasks in percentage) and a compound index of cognitive reserve. The cognitive reserve index was defined as the averaged z-score for the WAIS vocabulary subtest, National Adult Reading Test -NART- score, years of education. A compound indicator may provide a more precise measure of reserve than individual measures and limit potential collinearity issues (Jones et al., 2011). Such method was proven to attain good convergent and discriminant validity for cognitive reserve by Siedlecki et al., 2009.

Finally, interaction effects with age and connectivity metrics were also investigated by adding a second variable of interest (task accuracy, cognitive reserve index or education) in the model.

Measure of effect size. For all GLM, Cohen's $f^{2}$ (Cohen, 1988) was used to visualise spatial maps of the effect size of age on $\mathrm{FC}$, and compare them across tasks. The variant of Cohen's $f^{2}$ used here measured local effect size, i.e. the additional variance uniquely accounted by a variable of interest (age), expressed as a proportion of the variance unexplained by the model as a whole. This measure is useful for multivariate regression models including many variables (Selya, Rose, Dierker, Hedeker, \& Mermelstein, 2012).

Quantification of spatial maps similarities across and within cognitive states. We further investigated whether the effect of age on connectivity is largely driven by the cognitive state or rather stable across paradigms, using an analysis similar to Cole et al. (2014) and Geerligs et al. (2015). After averaging the connectivity matrices across participants within each cognitive state, a Pearson's correlation coefficient was used to quantify the similarity between age effect $\left(f^{2}\right)$ maps - for each ROI - in order to index the similarity in age-effect on $\mathrm{FC}$ across cognitive states. With the aim to further validate that the previous findings were robust, the intra-run reproducibility of age effect maps was additionally investigated. The internal consistency of the results was first tested by separating each available individual time series in two halves for each task, and repeating the GLM statistical analysis independently on each half (split-half reliability testing). Second, the impact of the block experimental design alternating periods of task and periods of rest - was tested: connectivity measures were generated using only time points collected within each block (IB), "purely" composed of task state. This step was not performed for the resting-state, which did not follow a block experimental design. We compared intra-run age effect maps using Pearson's correlation coefficients, as was done for between-state comparisons, both for the split-half and the within-block replication experiments.

Potential confounds. Sex was always included in our regression models as a confounding variable. The regression model initially did not include motion, i.e. $F D$, or a measure of brain atrophy, i.e. volume of cortico-spinal fluid relative to brain volume (RCSF). Both motion and atrophy are associated with aging, and may remove meaningful age-related variance. The impact of these additional confound regressors was assessed at the whole-connectome level by replicating the previous age-FC regression analysis with FD only, RCSF only, or both FD and RCSF. The volume of CSF was measured using partial volume effects of tissue types on the anatomical scan, and estimated with the trimmed minimum covariance determinant method (Tohka, Zijdenbos, \& Evans, 2004).

\subsection{Computational environment}

All analyses were executed in parallel on the "Guillimin" supercomputer (www.computecanada.ca), under CentOS. The datasets were preprocessed using the Neuro Imaging Analysis Kit (NIAK) niak-boss-0.18.1 container powered by singularity (http://singularity.lbl.gov/ docs-docker). The container includes an Ubuntu operating system, the pipeline system for Octave and Matlab (PSOM) version 1.2.1 (Bellec et al., 2012), Octave version 3.6.1 (http://gnu.octave.org) and the Minc toolkit version 0.3.18 (www.bic.mni.mcgill.ca/ServicesSoftware/ ServicesSoftwareMincToolKit).

\section{Results}

\subsection{Behavioural performance analysis}

Behavioural accuracy scores were computed for each experimental task. Cognitive data that was complete and within interquartile range is presented in Table 2 
for the whole sample, divided into three age groups to illustrate age trajectory in cognitive scores.

There was a significant correlation between age and accuracy across all neuropsychological and vocabulary tasks, ranging from small $(r<0.15)$ to large $(r>0.35)$ (Cohen, 1988). When controlling for sex and education (partial correlation), the effect of age remained significant for all tasks $(p<0.05)$. Increasing age was positively associated with performance for all vocabulary tasks (Antonyms: $r=0.2, n=258, p=0.002$; Synonyms: $r=0.3$, $n=253, p<0.001$; Picture Naming: $r=0.37, n=242, p<$ $0.001),{ }^{1}$ while the association was negative for other cognitive domains (Matrix: $r=-3, n=239, p<0.001$; Letter Number: $r=-0.2, n=244, p=0.000$; Digit Symbol: $r=-0.5, n=245, p=0.000$ ) (Figure 2).

\subsection{Mean sample effects on the brain functional connectome}

Average functional connectivity was first computed for the whole sample, while controlling for age, during tasks and rest (Figure 3). This preliminary step aimed at better discerning general FC organisation from age-specific effects. Mean connectivity maps revealed a consistent brain connectivity pattern across cognitive states. The IFG, PSTG and pMTG generally showed high connectivity levels with temporal regions and the premotor and ventrolateral prefrontal cortex. The language $\mathrm{ROI}$ also showed high connectivity with occipital visual cortices and ventral and posterior parietal areas, respectively involved in visuo-perceptual abilities and cognitive control (Christophel, Cichy, Hebart, \& Haynes, 2015). This pattern followed ventral and dorsal pathways traditionally described for language processes (Saur et al., 2008). Regions of the DMN were also highly correlated whether during tasks or during rest: the PCC exhibited a strong connectivity with prefrontal regions as well as with a large area surrounding the angular gyrus, and to a lesser extent with temporal regions, in accordance with previous reports (Greicius et al., 2003).

Main associations at the whole sample level were further investigated over the connectome for each independent variable (accuracy, education, cognitive reserve index). When considering the whole sample, few significant effects were found and those were diversely expressed. Brain regions in which effects were significant were rather heterogeneous, but all included to some extent portions of the DMN. Only during the synonyms

\section{a. Vocabulary tasks}
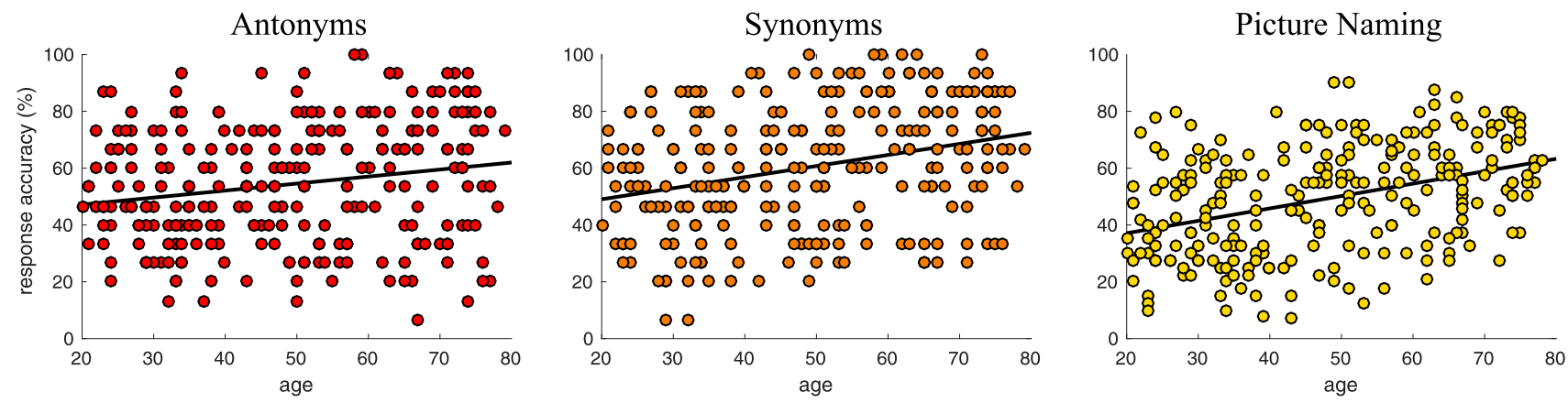

b. Neuropsychological tasks
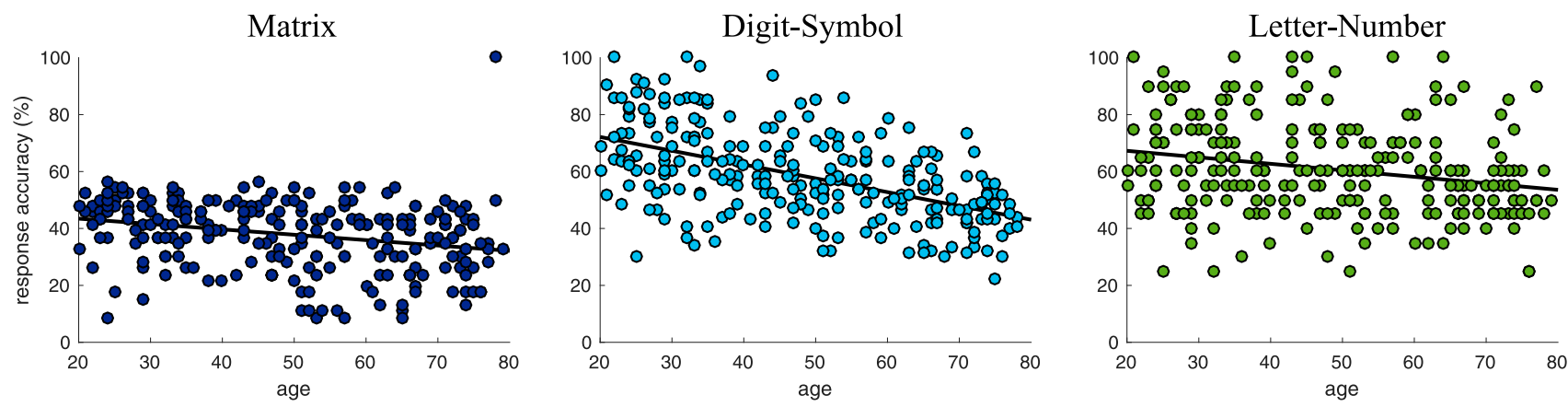

Figure 2. Behavioural regression plots for vocabulary and other cognitive tasks. In the upper row are the three vocabulary tasks (antonyms, synonyms, picture naming). In the bottom row are the three neuropsychological tasks (WAIS Matrix, Digit Symbol, Letter Number). For each, the graph shows the distribution of performance among the sample as well as the slope of the regression line. Vocabulary scores exhibit a significant and positive association with age, whereas the association is negative with neuropsychological scores. 


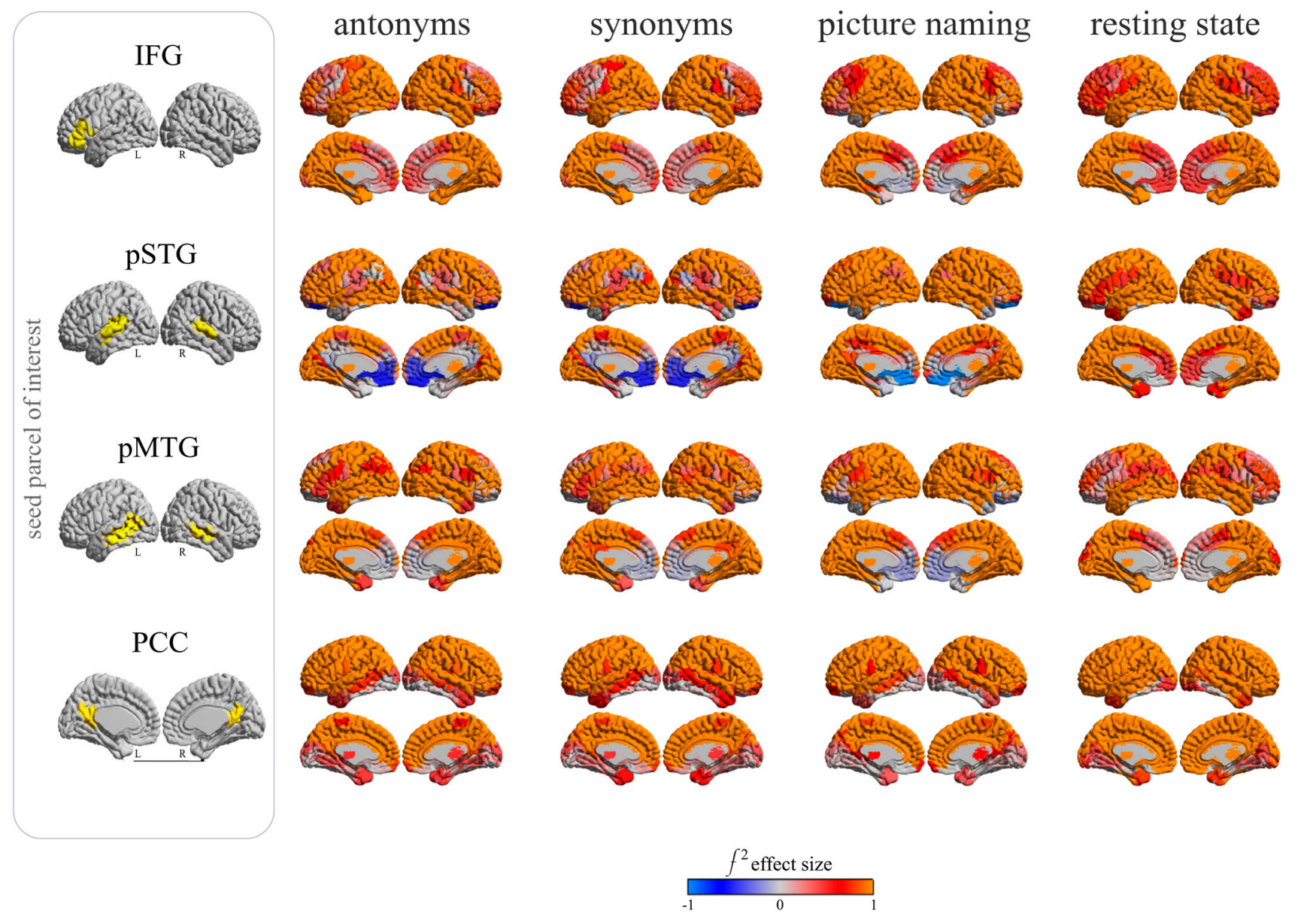

Figure 3. Average connectivity maps for each region of interest (ROI), during each cognitive state. Each map represents the $\mathrm{f} 2$ size of average connectivity between the seed region and all other regions in the brain (FDR correction $<0.05$, over the full connectome). The left column shows the localisation of the ROI: inferior frontal gyrus (IFG), posterior superior temporal gyrus (STG), posterior middle temporal gyrus (MTG) and posterior cingulate cortex. Each row shows the average connectivity of the designated ROI with the rest of the brain; and each column represent a different cognitive state, from left to right: antonyms, synonyms, picture naming and resting state.

task was there a significant association in FC with accuracy, in a large region including the cingulate cortex, the primary somatosensory cortex, the angular gyrus and the PMTG. The cognitive reserve index showed a significant main association during all cognitive states except the synonyms in regions of the posterior cingulate cortex, the angular gyrus, the primary motor cortex as well as the STG and MTG. Education alone was significantly associated with RS connectivity of the angular gyrus, the primary motor cortex, the primary somatosensory cortex, the ventral posterior part of the cingulate cortex, and the PSTG and not with other cognitive states.

\subsection{Effect of age on functional connectivity of the $D M N$ and language regions of interest}

A regression analysis, repeated across all region-toregion connections $(53 \times 53)$, showed mostly agerelated reductions in connectivity across cognitive states over the connectome. We investigated more specifically selected ROls in language and the DMN regions.

Association with age varied according to the $\mathrm{ROI}$ and effect sizes (FDR corrected $<0.05$, over the entire connectome) ranged from small $\left[f^{2}<0.15\right]$ to moderate $[0.15 \geq$ $f^{2}<0.35$ ] according to Cohen's guidelines (Cohen, 1988) (Figure 4). Fairly high similarity across all tasks was observed in the age effect connectivity maps of the pSTG and pMTG. By contrast, similarities were lower for the IFG when compared with other ROI.

The direction of the association was of special interest: language ROls exhibited an age-related increase in connectivity with the rest of the connectome, during tasks only. In contrast, the resting-state in general (across all ROIs) and the DMN in particular (across all cognitive states) exhibited only age-related decreases (Figure 4). Connectome-wide analyses confirmed that no significant age-related increases were seen outside of the language ROIs. Specifically, posterior MTG and STG both showed significant age-related increases in FC with occipital 


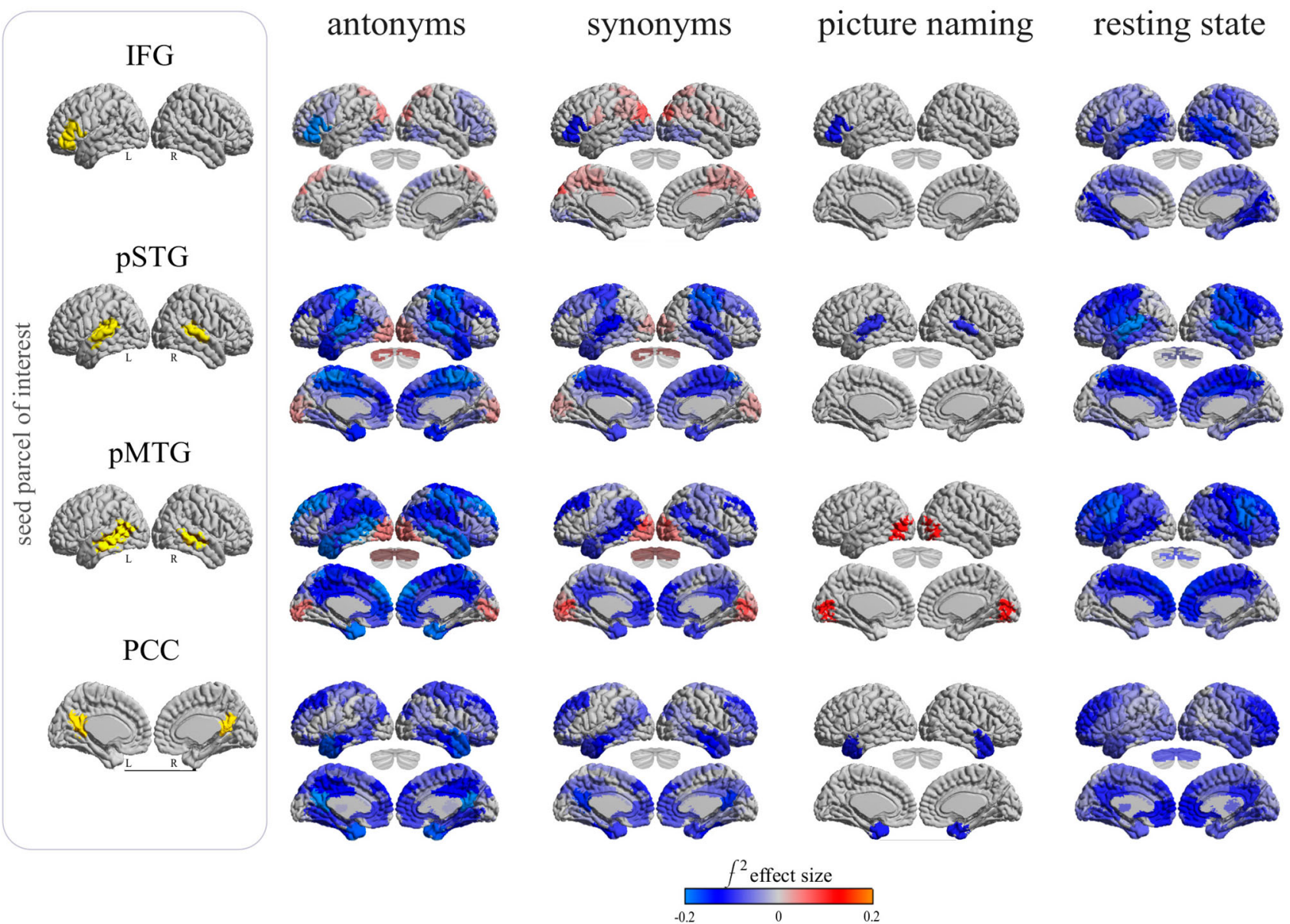

Figure 4. Age effect on cortical and cerebellar connectivity for each region of interest (ROI), for each cognitive state. Each map represents the $f 2$ size of age effects for the connectivity between the seed region and all other regions in the brain (FDR correction $<0.05$, over the full connectome). The left column shows the localisation of the ROI: inferior frontal gyrus (IFG), posterior superior temporal gyrus (STG), posterior middle temporal gyrus (MTG) and posterior cingulate cortex. Each row shows the changes in connectivity of the designated $\mathrm{ROI}$ with the rest of the connectome; and each column represent a different cognitive state, from left to right: antonyms, synonyms, picture naming and resting state. Regions of the language network exhibit significant age-related connectivity increases during vocabulary tasks. Resting state is conversely marked by a general decrease in connectivity as a function of age. Spatial connectivity patterns also vary from one region of the language network to another.

regions during language tasks. The IFG also showed an age-related increase in connectivity with parietal regions bilaterally, posterior to the central sulcus, in the angular and supramarginal gyrus and the parieto-occipital junction, during the antonyms and synonyms tasks. In contrast with connectivity increases for the language $\mathrm{ROIs}$, there was a global age-related decrease in DMN connectivity (seeded in the PCC) across all cognitive states.

\subsection{Interaction between age and other independent measures on the brain functional connectome}

Interaction effects were explored between the effect of age and other individual variables (task accuracy, cognitive reserve index, years of education) on connectivity. The only significant interaction was found between age and years of education, and exclusively during the RS. This interaction showed significant changes in connectivity in the form of a local increase in a bilateral fronto-parietal network (IFG, primary motor and somatosensory cortex, angular gyrus) as well as portions of the DMN (ventral posterior, dorsal and anterior cingulate cortex, angular gyrus). This result suggests that education influences the relationship between age and FC. No interaction was found between accuracy or the compound index of cognitive reserve and age-FC relationship.

\subsection{Age-effects similarities across cognitive states}

Previous visualisation of age effect maps (Figure 4) qualitatively revealed that the spatial expression of age effects varies from one cognitive state to another. The similarity in age effects between all cognitive states was further 
quantified. A correlation between the spatial maps of age effect for each ROls and each possible pair of cognitive state (whole run) was computed (Figure 5).

Correlation strength varied according to pairs of cognitive states ( $r=0.03-0.99$; see Figure 6$)$. Antonyms and synonyms, which are the two most cognitively similar tasks in this study, also had the highest similarity in terms of age effects (minimum $r=0.70$; maximum $r=$ 0.90). In other words, the effect of age on the connectivity of language and DMN regions was spatially similar during the completion of both tasks. Age effect in the antonyms and synonyms tasks were most dissimilar with RS. In comparison, age effect during picture naming was less similar to the latter tasks (minimum $r$ $=0.27$; maximum $r=0.78$ ). Nonetheless, age effect during picture naming had even more dissimilarity to rest (minimum $r=0.10$; maximum $r=0.47$ ).

\subsection{Robustness of age-effects within each cognitive state}

With the aim to validate that age effects on FC were mostly driven by the cognitive context rather than random noise or the design of the experimental paradigm, the intra-run reliability of age effect maps was assessed. Reproducibility testing was done first by replicating analyses after splitting the data into two halves, and, second, by analysing only data points within task blocks instead of the whole run. The previous conclusions (similarity levels in age effect between cognitive states when considering the whole runs) generalised to all models elaborated within cognitive state: within each cognitive state, a strong spatial correlation between age effect maps was observed when comparing split-half, intra-blocks and whole run models (Figure 7). The antonyms task, in particular, reached an almost perfect within-run correlation $(r=0.92,0.98,0.97$, 0.93 ) in age effects, for all ROI. These observations were confirmed visually, with highly similar spatial maps across models, in particular between split-halves (supplementary figure 2 ).

\subsection{Impact of confound parameters}

We systematically assessed at the whole-connectome level the impact of a number of covariates on age effects: sex, motion measured by frame displacement (FD), atrophy measured by relative corticospinal fluid (RCSF). A spatial Pearson's correlation was generated for each ROI, measuring the similarity between age effect maps derived from different models (age + sex,
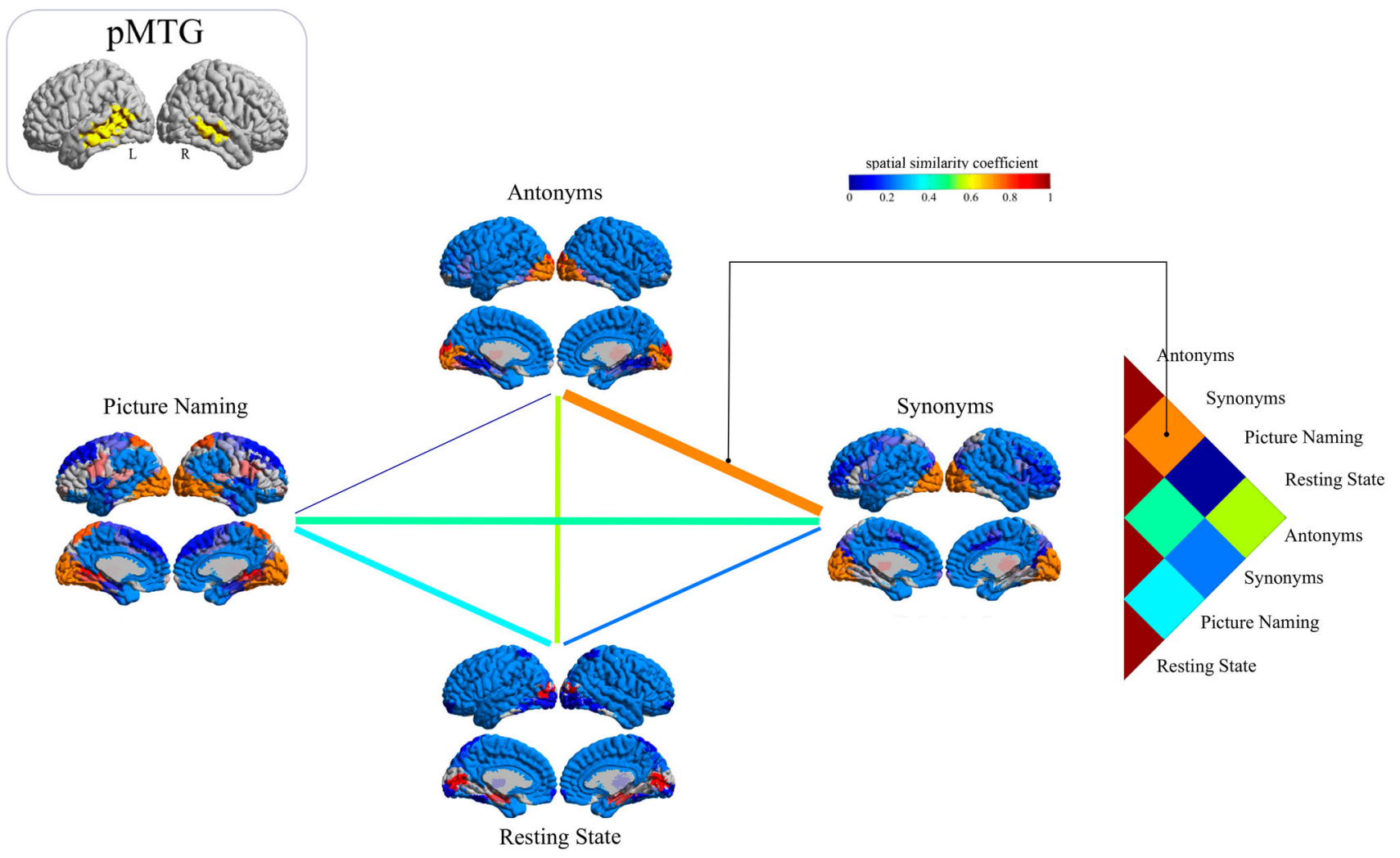

Figure 5. Illustration of the method used for comparison across cognitive states (whole run). The similarity in age effects were evaluated by correlating the statistical spatial maps for each pair of task, and for each ROI. Here is presented as an example the spatial similarity of spatial maps between each task for the posterior middle temporal gyrus (pMTG). The thickness and colour scale represent the strength of the correlation. For example, the functional connectivity spatial maps of the synonyms and antonyms tasks present high similarity $(r=0.83)$. 


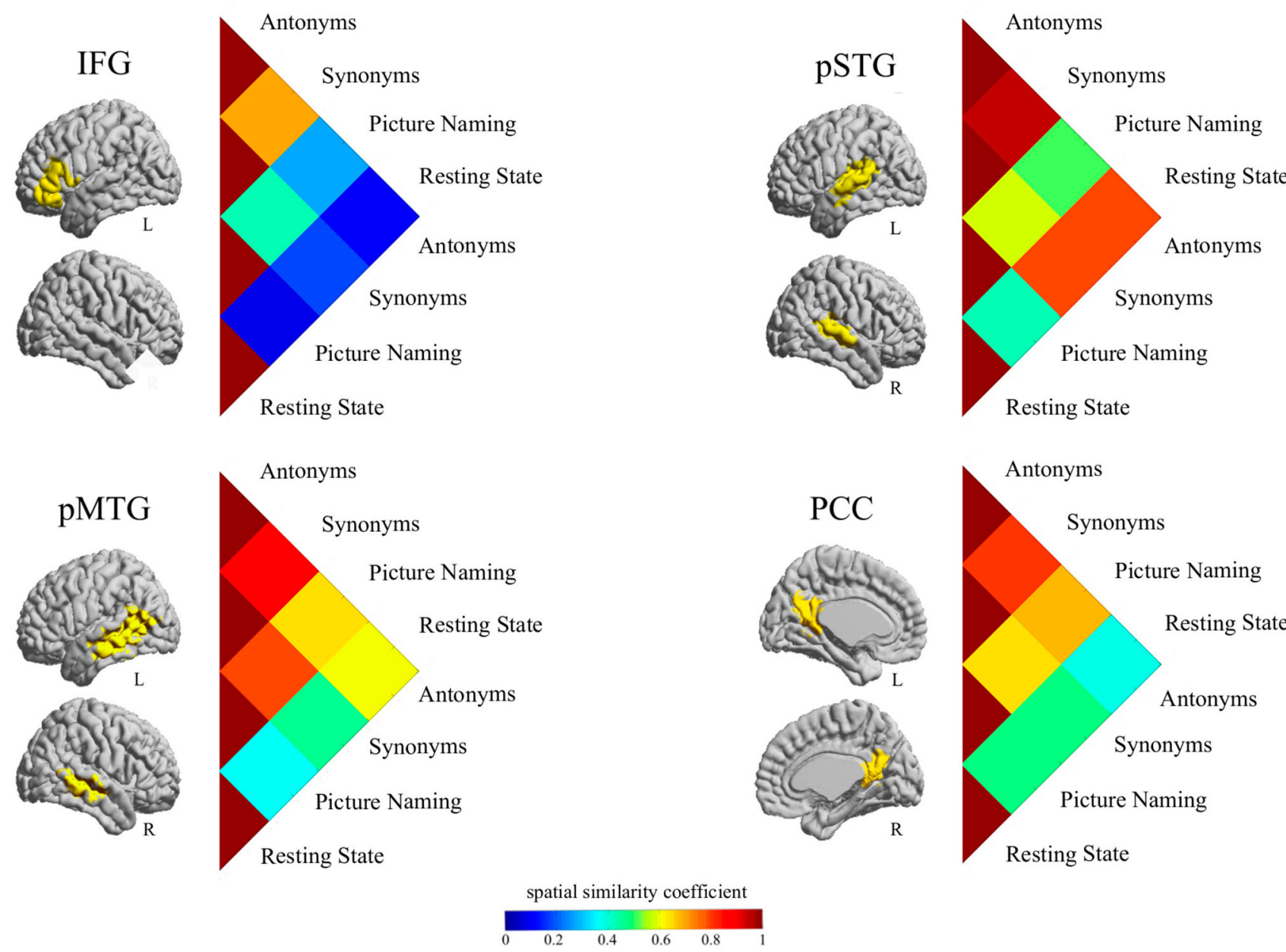

Figure 6. Inter task (whole run) age effect comparison. For each ROI (IFG, STG, MTG, PCC), r correlation matrix of effect sizes connectivity between cognitive states (antonyms; synonyms; picture naming; resting- state).

Note: the FC maps are not corrected for FDR, to allow for comparison between actual functional connectivity values. There is higher similarity in age effect between antonyms and synonyms tasks throughout regions of the language network. Age effect on brain connectivity during the resting state is generally less correlated to vocabulary tasks, although this pattern varies from seed to seed.

age + sex + FD, age + sex + RCSF, age + sex + FD + RCSF). The various models showed overall very high correlations. Only the simultaneous use of three covariates (age + sex + FD + RCSF) appeared to substantially impact the results but still showed a moderate association with other models (Figure 8). Cumulating all covariates in the model resulted in fewer discoveries: fewer brain regions were associated with age-related decreased functional connectivity. Local increases in connectivity of the language ROI persisted, even if there were also less numerous associations. We concluded that our findings are robust: additional confounds entered in the regression model do not modify the findings.

\section{Discussion}

The main goals of this study were to describe and compare age-related differences in functional connectivity between vocabulary tasks and resting state. While resting-state has been widely studied, vocabulary is an example of a preserved cognitive ability throughout the age trajectory. Secondary objectives included assessment of the association between age and accuracy for vocabulary and other cognitive tasks, as well as potential interactions with accuracy or cognitive reserve estimates. Results first confirmed that accuracy scores for semantic and vocabulary knowledge constantly improve throughout one's lifetime in our sample, in contrast to other cognitive domains. The concomitant FC patterns showed widespread age-related decreases in and between the language and defaultmode networks. These age-related FC decreases were observed both in RS and vocabulary tasks. Remarkably, age-related FC increases were found specifically for core language regions during vocabulary tasks. Yet, there was no evidence of an interaction between FC, age and task accuracy or cognitive reserve estimates, 


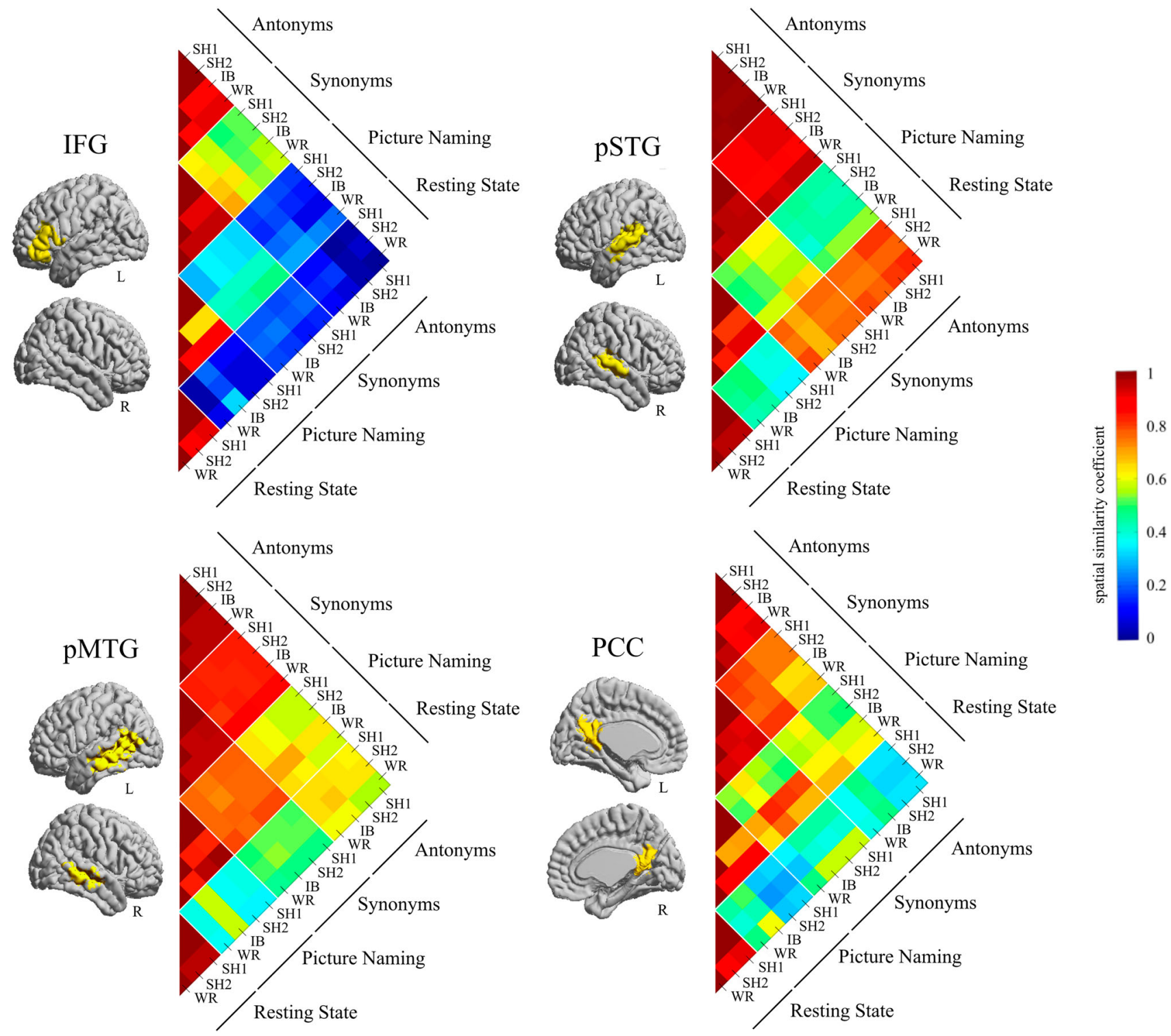

Figure 7. Intra and inter-task age effect comparison. For each ROI (IFG, pSTG, pMTG, PCC), correlation matrix ( $r$ correlation coefficient) between FC effect size maps within and across cognitive states (antonyms, synonyms, picture naming and resting state) for each GLM model investigated: split half validation (SH1 and SH2), intra-block connectivity (IB), and whole-run (WR).

Note: the FC matrices are not corrected for FDR, to allow for comparison between actual functional connectivity values. Intra-task, there is a strong spatial correlation within each task when comparing split-half, intra-blocks and whole run models.

challenging most cognitive models of aging. This study demonstrates that age-related differences in FC largely depend on the cognitive state. In other words, results derived from RS only do not systematically generalise to other cognitive states.

\subsection{The increase of vocabulary accuracy throughout age trajectory}

All vocabulary tasks exhibited a significant increase in accuracy with age. By contrast, age-related decreases in accuracy were found with fluid reasoning, perceptual speed and working memory tasks. These results are in line with prior reports (Diaz, Rizio, \& Zhuang, 2016; Fabiani, 2012; Kavé, Fridkin, \& Ayalon, 2018; Schaie \& Willis, 2010; Verhaeghen, 2003).

While the literature has reported a dissociation in age effects on reception and production abilities, the present vocabulary tasks were not designed to dissociate those aspects. In particular, the choice of constructs, linguistic properties of stimuli, as well as response modalities were not adapted to contrast reception and production. Both vocabulary reception and production tasks revealed an age-related increase in accuracy. Performance on the picture naming task may appear surprising, knowing that older adults typically complain of word finding and tip- 

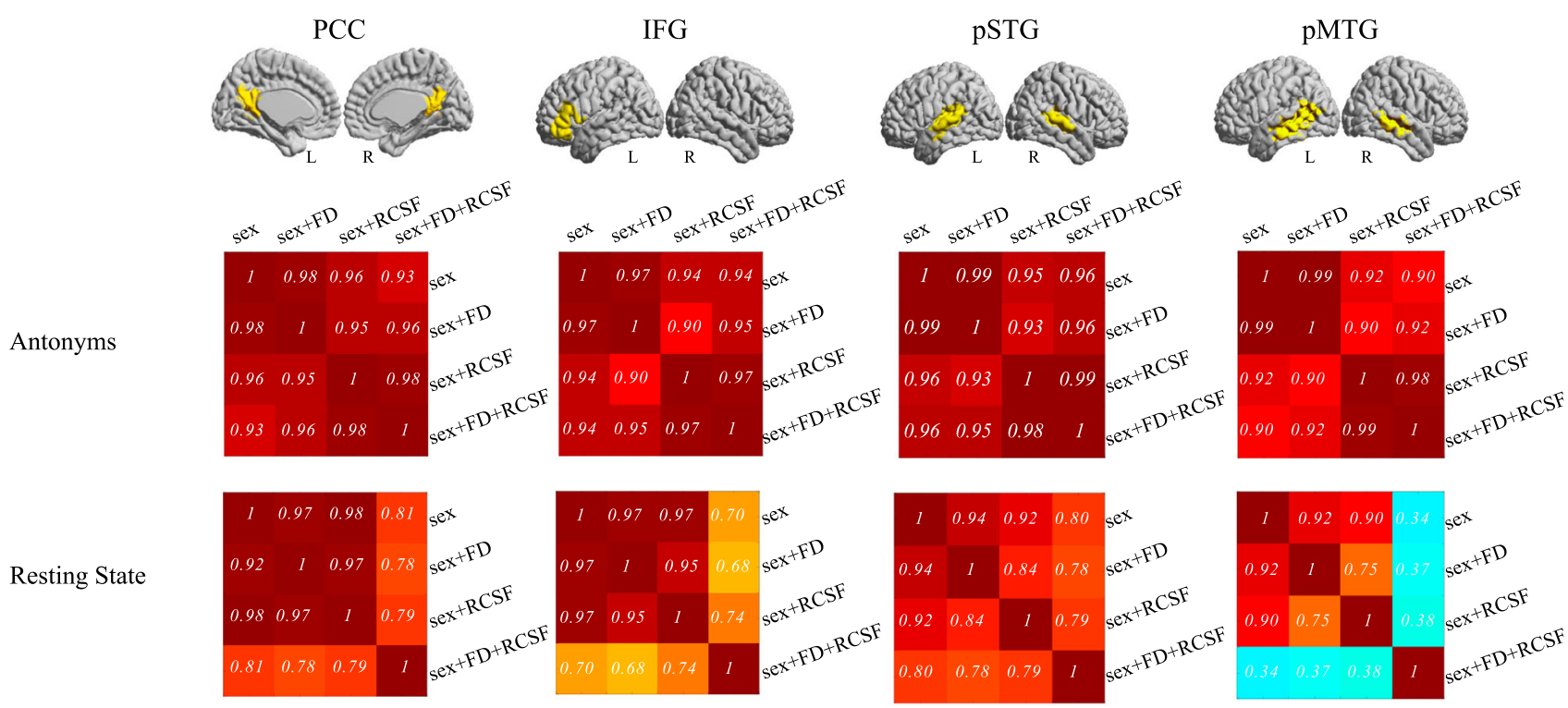

similarity coefficient

$\begin{array}{llll}0.2 & 0.4 & 0.6 & 0.8\end{array}$

Figure 8. Systematic comparison of potential confound parameters in the main GLM contrast: age-effect on the connectome. The matrix shows for two example cognitive states (antonyms and resting state) the correlation (Pearson's $r$ ) between effect sizes (f 2 ) of the various models tested: only sex included as a regressor, sex and frame displacement (FD), sex and relative cerebrospinal fluid (RCSF) or sex, frame displacement and relative cerebrospinal fluid altogether. There is high similarity between spatial maps obtained with each model, whether during the vocabulary tasks or during the resting-state task.

of-the-tongue problems in everyday life (Schwartz \& Frazier, 2005; Shafto, Burke, Stamatakis, Tam, \& Tyler, 2007). Inconsistent findings were previously reported in regards with picture naming performance. Those may in part be explained by methodological choices (Bowles \& Salthouse, 2008; Goulet, Ska, \& Kahn, 1994). The picture naming task was designed here to focus on vocabulary knowledge and, as such, did not disadvantage older individuals for four reasons. First, accuracy was used as a measure of performance, rather than response time, known to reflect general cognitive abilities (Shafto et al., 2016). Second, participants had $4.5 \mathrm{~s}$ to answer, limiting the impact of processing speed. Third, exceptions to the scoring criteria may have favoured individuals with slight phonological difficulties, but cases were rare. Finally, a one-word response was required, while word finding difficulties typically occur in the flow of a conversation (Schmitter-Edgecombe et al., 2000).

The vocabulary tasks used here have been reported to follow non-linear trajectories in accuracy within a larger sample (>4000), with an increase until age 65, followed either by a plateau, for synonyms, or a slight decrease, for antonyms and picture naming (Salthouse, 2014). Within the age range of the present study (18-80 y.o.), a clear and steady increase in accuracy was observed for all vocabulary tasks. They are thus particularly suitable to study potential brain mechanisms related to the preservation of cognition.

\subsection{Age-related functional connectivity changes in regions of the default mode network}

A widespread trend was a decrease in FC with age, observed both in vocabulary tasks and RS. This result extends - across all cognitive states and most regions the decrease within DMN connectivity with age commonly described at rest (see Sala-Llonch et al., 2015 for a review). This connectivity decrease across all cognitive states suggests a generalised disruption in grey and white matter integrity (Andrews-Hanna et al., 2007).

Age-related increases were previously reported in DMN inter-network connectivity, i.e. less segregation of the DMN with age, during tasks (Chan et al., 2017; Geerligs, Maurits, Renken, \& Lorist, 2014). Such increases appeared in tasks for which cognitive performance is declining with age and might thus depend on the level of task demand (Sambataro et al., 2010).

The DMN was initially described by subtracting BOLD activity levels of task and baseline epochs. Such "tasknegative" approach has since been criticised (see Callard \& Margulies, 2014), as it might conceal dynamic functional processes (Li, Wang, Yao, Hu, \& Friston, 2012) better characterised using FC analysis. 
The DMN indeed plays an active role in processes such as internalised thoughts (Spreng \& Grady, 2010) and of particular interest for this study - semantics (Humphreys, Hoffman, Visser, Binney, \& Lambon Ralph, 2015; Seghier, Fagan, \& Price, 2010; Wirth et al., 2011). The DMN and the networks activated by semantic tasks also partially overlap (Muller \& Meyer, 2014; Seghier et al., 2010). In sum, it might be hypothesised that vocabulary tasks do not generate large differences in DMN connectivity (Spreng \& Schacter, 2012), either because they do not highly challenge older individuals' capacity; or because they activate regions functionally related with the DMN. Unfortunately, the scarce number of studies exploring functional networks outside the DMN limits interpretation. With the aim of expanding upon the large majority of research that has focused on the DMN, this study additionally explores the impact of age on the connectivity of regions of the language network.

\subsection{Age-related functional connectivity changes in regions of the language network}

As observed with the DMN, connectivity between core language regions (inferior frontal gyrus, posterior middle and superior temporal gyrus) showed agerelated decreases. This observation contradicts our initial hypothesis. Previous work indeed reported increases within the fronto-temporal network (Agarwal, Stamatakis, Geva, \& Warburton, 2016; Meinzer et al., 2012), which are suspected to reflect top-down mechanism in support to cognitive performance (compensation). These two studies used fluency tasks and a phonological rhyming judgment task, respectively, that place more demand on executive and phonological processes than the word semantic tasks used here. Other abilities preserved with age, such as syntax comprehension, have not shown differential recruitment of neural networks in older adults, when task demand is controlled (Shafto \& Tyler, 2014).

The most striking result of this study was that core language regions increased their connectivity with regions outside the canonical language system, solely during the vocabulary tasks. Although effect sizes are of small amplitude (Figure 4), those increases truly contrast with the general decrease seen in the rest of the connectome and during RS.

First, during vocabulary tasks, temporal regions classically associated with the retrieval of semantic conceptual knowledge (Price, 2012) increased their connectivity with posterior (occipital and cerebellar) regions. The increase in temporo-occipital connectivity might suggest a higher reliance on networks specialised for the visual-semantic components of the task a strategy potentially beneficial to older adults. The left lateral and ventral visual cortex were indeed demonstrated to serve visual-semantic mechanisms in aging during visual object recognition (Park et al., 2004) and skilled reading (Price, 2012). Alternatively, the confound of perceptual abilities should not be underestimated in older adults (Monge \& Madden, 2016; Price \& Devlin, 2003; Salthouse, Hancock, Meinz, \& Hambrick, 1996). In this study, this potential confound was mitigated by providing $\mathrm{fMRI}$ compatible lenses, when needed. Yet these lenses do not achieve complete visual correction and might bring additional effort to complete the task. Challenges in low-level visual perception for older adults might thus be a straightforward explanation for the temporo-occipital connectivity increase with age. In turn, the increase in temporo-cerebellar connectivity is compatible with recent works on functional specialisation of cerebellar territories (Buckner, Krienen, \& Yeo, 2013): the more posterior lobules (VI, VII,IX) are thought to modulate cognition in general and language in particular (e.g. semantic judgment, verbal fluency, covert reading and higher linguistic levels, see Price, 2012 for a review). Because the cerebellum is still often unreported in $\mathrm{fMRI}$ studies, information remains scarce and further investigation will be required to better understand its rich interplay with cognition and aging (Bernard \& Seidler, 2014).

Second, the left IFG (Broca region) shows FC increases with parietal medial and posterior regions as well as superior posterior occipital regions. A diversity of information is conveyed across these two systems, including perception, attention, expectation and regulation. The IFG region was repeatedly suggested to be a binding point for neural compensation in aging, operating in support of regions engaged in the task in order to reach better performance (Cabeza \& Dennis, 2012; Park \& Reuter-Lorenz, 2009). The fronto-parietal increase in connectivity, for instance, may be evocative of a higher reliance on domain general abilities (Campbell \& Tyler, 2018). Directly testing the role of general cognitive abilities in aging would require a battery of tasks, including both preserved and declining abilities, as well as varying cognitive loads. This is an important line for future work, initiated by the RANN (Stern et al., 2014) and the Cambridge Centre for Aging and Neuroscience (Taylor et al., 2015) studies.

The interpretation of increases in connectivity as compensation, although tempting, needs to be explicitly tied to modulations in cognitive task performance (Steffener et al., 2014), as well as individual life experiences. 


\subsection{The association between performance, cognitive reserve estimates and brain connectivity}

The compensatory nature of a neural pattern needs to be supported by a three-way interaction: the higher the accuracy, the more connectivity would change as a function of age. Compensatory patterns should also exhibit recruitment of atypical functional resources. Contrary to the initial hypothesis, this study reports few associations between functional connectivity and other individual variables.

Results did not show the expected interaction between connectivity and accuracy scores in relation with aging, as was originally reported during RS (Andrews-Hanna et al., 2007; Damoiseaux et al., 2008; Jockwitz et al., 2017; Onoda, Ishihara, \& Yamaguchi, 2012). In fact, connectivity studies that used tasks tapping into preserved language abilities in aging (e.g. word production or syntax comprehension task) often similarly failed to find associations between brain function and performance scores (Agarwal et al., 2016; Chan et al., 2017; Davis, Zhuang, Wright, \& Tyler, 2014; Diaz, Rizio, \& Zhuang, 2016; Hoyau et al., 2018; La et al., 2016; Marsolais, Perlbarg, Benali, \& Joanette, 2014; Meunier, Stamatakis, \& Tyler, 2014; Tyler et al., 2010). This lack of association suggests that age-related changes tend to decrease when task complexity is controlled. Because semantic knowledge is generally maintained with age, older individuals on average might not reach their maximum capacity threshold and adapt the efficiency of their neural system to the task demand (Bartres-Faz \& Arenaza-Urquijo, 2011; Barulli, Rakitin, Lemaire, \& Stern, 2013; Persson et al., 2016; Steffener \& Stern, 2012).

Beyond performance, another goal of this study was to investigate if cognitive reserve - as assessed through education level alone or a compound index is associated with brain FC. Cognitive reserve is conceptualised as the potential to mitigate age-related brain decline and support cognitive performance (Steffener et al., 2011; Steffener et al., 2014; Stern, 2009). Existing evidence relating healthy aging and cognitive reserve is still scarce in $\mathrm{FMRI}$ - even more so in the FC literature and, to the best of our knowledge, the link between FC and cognitive reserve has never been investigated in the particular context of language abilities. Precise a priori hypotheses were consequently lacking regarding the relationship between age, cognitive reserve and FC. In this study, the main effect of the compound index of cognitive reserve on FC was detected in most cognitive states. No interaction was found between the compound index of cognitive reserve and age-FC relationship.
Conversely, the main effect of education on FC was only detected in RS, as well as a significant interaction between age and education on fronto-parietal FC. Education level might in fact critically influence the brain's ability to use more specialised functional networks (Marques et al., 2016) and was reported to associate with increases in bilateral fronto-parietal connectivity (Arenaza-Urquijo, 2013; Franzmeier, Buerger, et al., 2017; Perry et al., 2017). The lack of significant age-education interaction on FC during vocabulary tasks remains hard to interpret and warrants further exploration using tasks drawn from a variety of cognitive contexts.

\subsection{Age-effects dependence on the cognitive state}

The last contribution of this study lies in its comparison of age effects across vocabulary tasks and RS. RS is frequently presented as a fixed backbone that supports cognitive functioning (Ito et al., 2017) and, as such, could be considered as a stable individual "trait", generalisable to any cognitive state. When comparing RS and three vocabulary tasks, the effect of age on brain connectivity was clearly distinguishable in each cognitive context, with a tendency for more resemblance between similar paradigms (i.e. tasks relying on a motor response after semantic processing and word selection).

A split-half reliability analysis further demonstrated that age-effects maps are highly reproducible within that sample, rather than related to random noise. And secondly, the similarity of age effect maps between cognitive states cannot be explained by a simple model of response to the inherent timing of the task paradigm (within block model), contrary to previous propositions (Shah, Cramer, Ferguson, Birn, \& Anderson, 2016).

In contrast with what is observed during RS, and as discussed above, vocabulary tasks all exhibit localised increases in connectivity with age. Thus, even though average connectivity might be comparable across cognitive states (Cole et al., 2014; Mattar, Cole, ThompsonSchill, \& Bassett, 2015), cognitive states can reveal considerable inter-individual differences (Dixon et al., 2017; Finn et al., 2017; Geerligs et al., 2015; Mill, Ito, \& Cole, 2017). This is in line with the idea that RS, rather than a stable measure of individual cognitive traits, reflects a collection of various mental states (Allen et al., 2014; Dubois, 2016).

\subsection{Methodological considerations}

There are several general methodological limitations that should be taken into account when interpreting the 
present results. First, when contrasting cognitive states, the potential impact of movement cannot be overlooked. Vocabulary tasks, and in particular the overt picture naming task, suffered from more volumes suppressions than RS, thus limiting the number of frames and participants subjected to analysis, and negatively impacting statistical power. This may explain the lower number of connections exhibiting significant age effects, for the picture naming task in particular. Conversely, RS exhibited less movement in comparison with tasks, leading to a larger number of frames submitted to analysis, which might have in turn maximised the number of statistical discoveries. However, a scrubbing procedure was carefully implemented to reduce motion artefacts and the effect of including movement as a covariate in our GLM model was negligible.

The statistical correction applied for multiple comparisons in whole-brain FC studies might also limit the number of positive discoveries for association with cognitive performance. For example, Geerligs in 2015 mentions that the correlation obtained between behaviour and FC graph-measures did not survive FDR correction and should therefore be interpreted with caution. While univariate correlation analysis used in the present study is beneficial to the understanding of linear relationships between age and functional coupling between brain regions, other methods such as intra agegroup univariate analysis (Andrews-Hanna et al., 2007; Jockwitz et al., 2017; Stern, Gazes, Razlighi, Steffener, \& Habeck, 2018), dynamic functional connectivity (Cabral et al., 2017; Shine et al., 2016) or multivariate methods (Battaglia et al., 2017; Samu et al., 2017; Schultz \& Cole, 2016) have been pointed out as having a strongest potential to qualify individual heterogeneity. Additionally, future explorations of the cognitive and neural reserve might further benefit from more precise manipulation of the task paradigm (as described in length in Mill et al., 2017), using more complex conditions (Stanley, Dagenbach, Lyday, Burdette, \& Laurienti, 2014) or tapping both maintained and declining abilities to reach the "efficiency" threshold.

Participant selection was another critical aspect of the present large-sample study. Out of 300, only 113 participants remained when including those with complete data after quality control, across the whole range of neuroimaging and behavioural tasks. Considering this large attrition, all subjects with available data for a given contrast were included in statistical test with the aim of maximising statistical power (Bhattacharjee, Dhar, \& Subramanian, 2011). High statistical power was particularly important in the initial phase of connectome-wide exploration of age-related connectivity during vocabulary tasks.
Finally, but importantly, the interpretation of increases or decreases in connectivity remains an open question in the literature. Based on a univariate model alone, it is impossible to deduce the underlying neural action, because both activation or deactivation are theoretically possible. For example, this study cannot conclude whether top-down or bottom-up processes occur between frontal and posterior regions. Connectivity decreases are suspected to affect the ability to transfer information between brain areas, thus hindering cognitive performance (Damoiseaux et al., 2008; Geerligs, Saliasi, Renken, Maurits, \& Lorist, 2014; Onoda et al., 2012). In this view, only tasks that show performance decline with age would reveal decreases in connectivity of task-related regions, as well as decreased coupling with default mode activity (Samu et al., 2017). Conversely, a decrease in functional connectivity can be interpreted dramatically differently, as enhanced efficiency and proficiency. This was evidenced, for example, in motor (Doyon et al., 2009) or second language learning (Ghazi Saidi et al., 2013). The present study does not allow us to conclude which of these distinctive interpretations is the right one, but since older participants do achieve a performance level that is comparable to that of younger adults, the idea of a more efficient functional network is a very attractive one. In this view, a decrease in $\mathrm{FC}$ between core language regions would reflect more cost-effective communication (Di \& Biswal, 2018), which would parallel the development of vocabulary knowledge throughout one's lifetime. In turn, age-related increases in the connectivity of language regions with posterior areas could hypothetically reflect less efficient sensory and attentional processes.

\section{Conclusion}

This study highlights that distinct information is provided by task-based and resting-state designs in the exploration of the impact of aging on the brain functional connectivity. Using a seed-based and connectome-wide approach, results suggest that preserved vocabulary knowledge do not abide to traditional models of cognitive aging that were developed from other domains, or to the snapshot offered by RS. The implications are that neuroscience of aging should not ignore the effect of the specific cognitive context under study. The resting state itself is not homogeneous, but rather constitutes another experimental condition, composed of a mixture of many uncontrolled cognitive states (Gonzalez-Castillo et al., 2015; Shah et al., 2016) and as such should not be considered as independent "trait" (Geerligs \& Tsvetanov, 2017). Acquired world knowledge through word semantic constructions - stands very 
peculiarly among human cognitive abilities and may help support the preservation of our role in society, without regards to age trajectory. Specifically, increases in connectivity between language regions and posterior brain areas during vocabulary tasks may hypothetically reflect an increased ability to take advantage of the semantic knowledge acquired throughout one's life, without the need to rely on compensatory mechanisms. Cognitive models of aging should thus be expanded in the future to encompass both losses and gains in cognitive performance, within a perspective geared towards multifaceted functional mechanisms.

\section{Note}

1. Response times were available for the antonyms and synonyms tasks only. For those two tasks, the relationship with age was not significant (Antonyms: $r=-0.08$, $n=258, p=0.22$; Synonyms: $r=-0.07, n=253, p=0.24$ ).

\section{Disclosure statement}

No potential conflict of interest was reported by the authors.

\section{Funding}

This work was supported in part by the National Institute on Aging grant RF1 AG038465 to Yaakov Stern and Christian Habeck.

\section{References}

Ad-Dab'bagh, Y., Lyttelton, O., Muehlboeck, J. S., Lepage, C., Einarson, D., Mok, K., ... Fombonne, E. (2006). The CIVET image-processing environment: A fully automated comprehensive pipeline for anatomical neuroimaging research. In Proceedings of the 12th annual meeting of the organization for human brain mapping (pp. 2266). Florence: OHBM.

Agarwal, S., Stamatakis, E. A., Geva, S., \& Warburton, E. A. (2016). Dominant hemisphere functional networks compensate for structural connectivity loss to preserve phonological retrieval with aging. Brain and Behavior, 6(9), e00495. doi:10.1002/ brb3.495

Allen, E. A., Damaraju, E., Plis, S. M., Erhardt, E. B., Eichele, T., \& Calhoun, V. D. (2014). Tracking whole-brain connectivity dynamics in the resting state. Cerebral Cortex, 24(3), 663-676.

Anderson, L. A., Day, K. L., Beard, R. L., Reed, P. S., \& Wu, B. (2009). The public's perceptions about cognitive health and Alzheimer's disease among the U.S. population: A national review. The Gerontologist, 49(S1), S3-S11. doi:10.1093/ geront/gnp088

Andrews-Hanna, J. R., Snyder, A. Z., Vincent, J. L., Lustig, C., Head, D., Raichle, M. E., \& Buckner, R. L. (2007). Disruption of large-scale brain systems in advanced aging. Neuron, 56 (5), 924-935. doi:10.1016/j.neuron.2007.10.038
Arenaza-Urquijo. (2013). Relationships between years of education and gray matter volume, metabolism and functional connectivity in healthy elders. Neuroimage, 83, 450-457.

Bartres-Faz, D., \& Arenaza-Urquijo, E. M. (2011). Structural and functional imaging correlates of cognitive and brain reserve hypotheses in healthy and pathological aging. Brain Topography, 24(3-4), 340-357. doi:10.1007/s10548011-0195-9

Barulli, D. J., Rakitin, B. C., Lemaire, P., \& Stern, Y. (2013). The influence of cognitive reserve on strategy selection in normal aging. Journal of the International Neuropsychological Society, 19(07), 841-844. doi:10.1017/ S1355617713000593

Battaglia, D., Thomas, B., Hansen, E. C., Chettouf, S., Daffertshofer, A., McIntosh, A. R., ... Jirsa, V. (2017). Functional connectivity dynamics of the resting state across the human adult lifespan. BioRxiv, 107243, doi:10. $1101 / 107243$

Bellec, P. (2013). Mining the hierarchy of resting-state brain networks: Selection of representative clusters in a multiscale structure. In International workshop on pattern recognition in neuroimaging (pp. 54-57). IEEE. doi:10.1109/PRNI. 2013.23

Bellec, P., Benhajali, Y., Carbonell, F., Dansereau, C., Albouy, G., Pelland, M., ... Orban, P. (2015). Impact of the resolution of brain parcels on connectome-wide association studies in fMRI. Neurolmage, 123, 212-228. doi:10.1016/j.neuroimage. 2015.07.071

Bellec, P., Lavoie-Courchesne, S., Dickinson, P., Lerch, J. P., Zijdenbos, A. P., \& Evans, A. C. (2012). The pipeline system for Octave and Matlab (PSOM): a lightweight scripting framework and execution engine for scientific workflows. Frontiers in Neuroinformatics, 6(April), 1-18. doi:10.3389/fninf.2012. 00007

Bellec, P., Perlbarg, V., Jbabdi, S., Pélégrini-Issac, M., Anton, J.-L., Doyon, J., \& Benali, H. (2006). Identification of large-scale networks in the brain using fMRI. Neuroimage, 29(4), 1231-1243.

Bellec, P., Rosa-Neto, P., Lyttelton, O. C., Benali, H., \& Evans, A. C. (2010). Multi-level bootstrap analysis of stable clusters in resting-state fMRI. Neurolmage, 51(3), 1126-1139. doi:10. 1016/j.neuroimage.2010.02.082

Benjamini, Y., \& Yekutieli, D. (2001). The control of the false discovery rate in multiple testing under dependency. Annals of Statistics, 29(4), 1165-1188.

Bernard, J. A., \& Seidler, R. D. (2014). Moving forward: Age effects on the cerebellum underlie cognitive and motor declines. Neuroscience and Biobehavioral Reviews, 42, 193207. doi:10.1016/j.neubiorev.2014.02.011

Bhattacharjee, M., Dhar, S. K., \& Subramanian, S. (2011). Recent advances in biostatistics: False discovery rates, survival analysis, and related topics (Vol. 4). World Scientific.

Binder, J. R., Desai, R. H., Graves, W. W., \& Conant, L. L. (2009). Where is the semantic system? A critical review and metaanalysis of 120 functional neuroimaging studies. Cerebral Cortex, 19(12), 2767-2796. doi:10.1093/cercor/bhp055

Birn, R. M., Diamond, J. B., Smith, M. A., \& Bandettini, P. A. (2006). Separating respiratory-variation-related fluctuations from neuronal-activity-related fluctuations in fMRI. Neurolmage, 31(4), 1536-1548. doi:10.1016/j.neuroimage.2006.02.048

Bowles, R. P., \& Salthouse, T. A. (2008). Vocabulary test format and differential relations to age. Psychology and Aging, 23 (2), 366-376. 
Bozzali, M., Dowling, C., Serra, L., Spanó, B., Torso, M., Marra, C., ... Cercignani, M. (2015). The impact of cognitive reserve on brain functional connectivity in Alzheimer's disease. Journal of Alzheimer's Disease, 44(1), 243-250. doi:10.3233/JAD141824

Buckner, R. L., Andrews-Hanna, J. R., \& Schacter, D. L. (2008). The brain's default network. Annals of the New York Academy of Sciences, 1124(1), 1-38.

Buckner, R. L., Krienen, F. M., \& Yeo, B. T. T. (2013). Opportunities and limitations of intrinsic functional connectivity MRI. Nature Publishing Group, 16(7), 832-837. doi:10. 1038/nn.3423

Cabeza, R., \& Dennis, N. a. (2012). Frontal lobes and aging. Principles of Frontal Lobe Function, 2, 628-652. doi:10.1093/ acprof:oso/9780195134971.001.0001

Cabral, J., Vidaurre, D., Marques, P., Magalhães, R., Moreira, P. S., Soares, J. M., ... Kringelbach, M. L. (2017). Cognitive performance in healthy older adults relates to spontaneous switching between states of functional connectivity during rest. Scientific Reports, 7(1), 5135.

Callard, F., \& Margulies, D. S. (2014). What we talk about when we talk about the default mode network. Frontiers in Human Neuroscience, 8, 619. doi:10.3389/fnhum.2014.00619

Campbell, K. L., \& Schacter, D. L. (2017). Ageing and the resting state: Is cognition obsolete? Language, Cognition and Neuroscience, 32(6), 661-668.

Campbell, K. L., \& Tyler, L. K. (2018). Language-related domainspecific and domain-general systems in the human brain. Current Opinion in Behavioral Sciences, 21, 132-137. doi:10. 1016/j.cobeha.2018.04.008

Cao, M., Wang, J. H., Dai, Z. J., Cao, X. Y., Jiang, L. L., Fan, F. M., ... $\mathrm{He}$, Y. (2014). Topological organization of the human brain functional connectome across the lifespan. Developmental Cognitive Neuroscience, 7(16), 76-93. doi:10.1016/j.dcn.2013. 11.004

CDC. (2013). Self-reported increased confusion or memory loss and associated functional difficulties among adults aged $\geq 60$ years-21 states. Morbidity and Mortality Weekly Report, 62 (18), 347-350.

Chan, M. Y., Alhazmi, F. H., Park, D. C., Savalia, N. K., \& Wig, G. S. (2017). Resting-state network topology differentiates task signals across the adult life Span. The Journal of Neuroscience: The Official Journal of the Society for Neuroscience, 37(10), 2734-2745. doi:10.1523/JNEUROSCI. 2406-16.2017

Chételat, G., Landeau, B., Salmon, E., Yakushev, I., Bahri, M. A., Mézenge, F., ... Fellgiebel, A. (2013). Relationships between brain metabolism decrease in normal aging and changes in structural and functional connectivity. Neurolmage, 76, 167-177. doi:10.1016/j.neuroimage.2013.03.009

Christophel, T. B., Cichy, R. M., Hebart, M. N., \& Haynes, J.-D. (2015). Parietal and early visual cortices encode working memory content across mental transformations. Neurolmage, 106, 198-206. doi:10.1016/J.NEUROIMAGE.2014.11.018

Ciric, R., Wolf, D. H., Power, J. D., Roalf, D. R., Baum, G. L., Ruparel, K., ... Davatzikos, C. (2017). Benchmarking of participantlevel confound regression strategies for the control of motion artifact in studies of functional connectivity. Neuroimage, 154, 174-187.

Cohen, J. (1988). Statistical power analysis for the behavioral sciences. Hillsdale, NJ: Law-rence Erlbaum Associates. Inc, Publishers.
Cole, M. W., Bassett, D. S., Power, J. D., Braver, T. S., \& Petersen, S. E. (2014). Intrinsic and task-evoked network architectures of the human brain. Neuron, 83(1), 238-251. doi:10.1016/j. neuron.2014.05.014

Collins, D. L., Neelin, P., Peters, T. M., \& Evans, A. C. (1994). Automatic 3D intersubject registration of MR volumetric data in standardized Talairach space. Journal of Computer Assisted Tomography, 18(2), 192-205.

Damoiseaux, J. S., Beckmann, C. F., Arigita, E. J. S., Barkhof, F., Scheltens, P., Stam, C. J., ... Rombouts, S. A. R. B. (2008). Reduced resting-state brain activity in the "default network" in normal aging. Cerebral Cortex, 18(8), 18561864. doi:10.1093/cercor/bhm207

Davis, S. W., Stanley, M. L., Moscovitch, M., \& Cabeza, R. (2017). Resting-state networks do not determine cognitive function networks: A commentary on Campbell and Schacter (2016). Language, Cognition and Neuroscience, 32(6), 669-673.

Davis, S. W., Zhuang, J., Wright, P., \& Tyler, L. K. (2014). Agerelated sensitivity to task-related modulation of languageprocessing networks. Neuropsychologia, 63, 107-115. doi:10.1016/j.neuropsychologia.2014.08.017

Di, X., \& Biswal, B. B. (2018). Toward task Connectomics: Examining whole-brain task modulated connectivity in different task domains. Cerebral Cortex, doi:10.1093/cercor/ bhy055

Diaz, M. T., Rizio, A. A., \& Zhuang, J. (2016). The neural language systems that support healthy aging: Integrating function, structure, and behavior. Language and Linguistics Compass, 10(7), 314-334. doi:10.1111/lnc3.12199

Dixon, M. L., Andrews-Hanna, J. R., Spreng, R. N., Irving, Z. C., Mills, C., Girn, M., \& Christoff, K. (2017). Interactions between the default network and dorsal attention network vary across default subsystems, time, and cognitive states. Neurolmage, 147, 632-649. doi:10.1016/J.NEUROIMAGE. 2016.12.073

Dong, L., Shen, Y., Lei, X., Luo, C., Qing-Wei, L., Wu, W.-Y., ... Chun-Bo, L. (2012). The heterogeneity of aging brain: Altered functional connectivity in default mode network in older adults during verbal fluency tests. Chinese Medical Journal, 125(4), 604-610. doi:10.3760/cma.j.issn.0366-6999. 2012.04.009

Doyon, J., Bellec, P., Amsel, R., Penhune, V., Monchi, O., Carrier, J., ... Benali, H. (2009). Contributions of the basal ganglia and functionally related brain structures to motor learning. Behavioural Brain Research, 199(1), 61-75. doi:10.1016/J. BBR.2008.11.012

Dubois, J. (2016). Brain age: A state-of-mind? On the stability of functional connectivity across behavioral states. The Journal of Neuroscience: The Official Journal of the Society for Neuroscience, 36(8), 2325-2328. doi:10.1523/JNEUROSCI. 4312-15.2016

Fabiani, M. (2012). It was the best of times, it was the worst of times: A psychophysiologist's view of cognitive aging. Psychophysiology, 49(3), 283-304. doi:10.1111/j.1469-8986. 2011.01331.x

Finn, E. S., Scheinost, D., Finn, D. M., Shen, X., Papademetris, X., \& Constable, R. T. (2017). Can brain state be manipulated to emphasize individual differences in functional connectivity? Neurolmage, 160, 140-151. doi:10.1016/J.NEUROIMAGE.2017. 03.064

Fonov, V., Evans, A. C., Botteron, K., Almli, C. R., McKinstry, R. C., Collins, D. L., \& Group, B. D. C. (2011). Unbiased average age- 
appropriate atlases for pediatric studies. Neuroimage, 54(1), 313-327.

Franzmeier, N., Buerger, K., Teipel, S., Stern, Y., Dichgans, M., Ewers, M., \& A. D. N. I. (2017). Cognitive reserve moderates the association between functional network anti-correlations and memory in $\mathrm{MCl}$. Neurobiology of Aging, 50, 152-162.

Franzmeier, N., Hartmann, J. C., Taylor, A. N. W., Caballero, MÁA, Simon-Vermot, L., Buerger, K., ... Ewers, M. (2017). Left frontal hub connectivity during memory performance supports reserve in aging and mild cognitive impairment. Journal of Alzheimer's Disease, 59(4), 1381-1392. doi:10.3233/JAD170360

Garcia-Garcia, M., Nikolaidis, A., Bellec, P., Craddock, R. C., Cheung, B., Castellanos, F. X., \& Milham, M. P. (2018). Detecting stable individual differences in the functional organization of the human basal ganglia. Neurolmage, 170, 68-82.

Geerligs, L., Maurits, N. M., Renken, R. J., \& Lorist, M. M. (2014). Reduced specificity of functional connectivity in the aging brain during task performance. Human Brain Mapping, 35 (1), 319-330. doi:10.1002/hbm.22175

Geerligs, L., Renken, R. J., Saliasi, E., Maurits, N. M., \& Lorist, M. M. (2015). A brain-wide study of age-related changes in functional connectivity. Cerebral Cortex, 25(7), 1987-1999. doi:10.1093/cercor/bhu012

Geerligs, L., Rubinov, M., Cam-CAN, \& Henson, R. N. (2015). State and trait components of functional connectivity: Individual differences vary with mental state. The Journal of Neuroscience, 35(41), 13949-13961.

Geerligs, L., Saliasi, E., Renken, R. J., Maurits, N. M., \& Lorist, M. M. (2014). Flexible connectivity in the aging brain revealed by task modulations. Human Brain Mapping, 35(8), 3788-3804. doi:10.1002/hbm.22437

Geerligs, L., \& Tsvetanov, K. A. (2017). The use of resting state data in an integrative approach to studying neurocognitive ageing - commentary on Campbell and Schacter (2016). Language, Cognition and Neuroscience, 32(6), 684-691. doi:10.1080/23273798.2016.1251600

Ghazi Saidi, L., Perlbarg, V., Marrelec, G., Pélégrini-Issac, M., Benali, H., \& Ansaldo, A.-I. (2013). Functional connectivity changes in second language vocabulary learning. Brain and Language, 124(1), 56-65. doi:10.1016/j.bandl.2012.11. 008

Giove, F., Gili, T., lacovella, V., Macaluso, E., \& Maraviglia, B. (2009). Images-based suppression of unwanted global signals in resting-state functional connectivity studies. Magnetic Resonance Imaging, 27(8), 1058-1064.

Gonzalez-Castillo, J., Hoy, C. W., Handwerker, D. a., Robinson, M. E., Buchanan, L. C., Saad, Z. S., \& Bandettini, P. A. (2015). Tracking ongoing cognition in individuals using brief, whole-brain functional connectivity patterns. Proceedings of the National Academy of Sciences, 112(28), 8762-8767. doi:10.1073/pnas.1501242112

Goulet, P., Ska, B., \& Kahn, H. J. (1994). Is there a decline in picture naming with advancing age? Journal of Speech, Language, and Hearing Research, 37(3), 629-644. doi:10. 1044/jshr.3703.629

Goulet, P., Ska, B., \& Kahn, H. J. (1994). Is there a decline in picture naming with advancing age? Journal of Speech and Hearing Research, 37(June 1994), 629-644. doi:10.1044/jshr. 3703.629
Grady, C. L. (2016). Age differences in functional connectivity at rest and during cognitive tasks. In Cognitive neuroscience of aging (pp. 105-130). Oxford University Press. doi:10.1093/ acprof:oso/9780199372935.003.0005

Greicius, M. D., Krasnow, B., Reiss, A. L., \& Menon, V. (2003). Functional connectivity in the resting brain: A network analysis of the default mode hypothesis. Proceedings of the National Academy of Sciences of the United States of America, 100(1), 253-258. doi:10.1073/pnas.0135058100

Guzzetti, S., \& Daini, R. (2014). Inter-hemispheric recruitment as a function of task complexity, age and cognitive reserve. Neuropsychology, Development, and Cognition. Section $B$, Aging, Neuropsychology and Cognition, 21(6), 722-745. doi:10.1080/13825585.2013.874522

Hagmann, P., Cammoun, L., Gigandet, X., Meuli, R., Honey, C. J., Wedeen, V. J., \& Sporns, O. (2008). Mapping the structural core of human cerebral cortex. PLoS Biology, 6(7), e159.

Haimovici, A., Tagliazucchi, E., Balenzuela, P., \& Laufs, H. (2017). On wakefulness fluctuations as a source of BOLD functional connectivity dynamics. Scientific Reports, 7(1), 5908. doi:10. 1038/s41598-017-06389-4

Hillenbrand, S. F., Ivry, R. B., \& Schlerf, J. E. (2016). Impact of taskrelated changes in heart rate on estimation of hemodynamic response and model fit. Neurolmage, 132, 455-468. doi:10. 1016/J.NEUROIMAGE.2016.02.068

Hoyau, E., Roux-Sibilon, A., Boudiaf, N., Pichat, C., Cousin, E., Krainik, A., ... Baciu, M. (2018). Aging modulates fronto-temporal cortical interactions during lexical production. A dynamic causal modeling study. Brain and Language, 184, 11-19. doi:10.1016/j.bandl.2018.06.003

Humphreys, G. F., Hoffman, P., Visser, M., Binney, R. J., \& Lambon Ralph, M. A. (2015). Establishing task- and modality-dependent dissociations between the semantic and default mode networks. Proceedings of the National Academy of Sciences of the United States of America, 112(25), 78577862. doi:10.1073/pnas.1422760112

lordan, A. D., \& Reuter-lorenz, P. A. (2016). Age-related change and the predictive value of the "resting state": a commentary on Campbell and Schacter (2016). Language, Cognition and Neuroscience, O(0), 1-4. doi:10.1080/23273798.2016.1242759

Ito, T., Kulkarni, K. R., Schultz, D. H., Mill, R. D., Chen, R. H., Solomyak, L. I., \& Cole, M. W. (2017). Cognitive task information is transferred between brain regions via restingstate network topology. BioRxiv, 8(1), 10-27.

Jackson, R. L., Hoffman, P., Pobric, G., \& Lambon Ralph, M. A. (2015). The nature and neural correlates of semantic association versus conceptual similarity. Cerebral Cortex, 25(11), 4319-4333. doi:10.1093/cercor/bhv003

Jockwitz, C., Caspers, S., Lux, S., Eickhoff, S. B., Jütten, K., Lenzen, S., ... Amunts, K. (2017). Influence of age and cognitive performance on resting-state brain networks of older adults in a population-based cohort. Cortex, 89, 28-44. doi:10.1016/j. cortex.2017.01.008

Jones, R. N., Manly, J., Glymour, M. M., Rentz, D. M., Jefferson, A. L., \& Stern, Y. (2011). Conceptual and measurement challenges in research on cognitive reserve. Journal of the International Neuropsychological Society, 17(04), 593-601. doi:10.1017/S1355617710001748

Kavé, G., Fridkin, S., \& Ayalon, L. (2018). Demographic factors and retrieval of object and proper names after age 70 . PLOS ONE, 13(1), e0191876. doi:10.1371/journal.pone. 0191876 
Kong, R., Li, J., Orban, C., Sabuncu, M. R., Liu, H., Schaefer, A., ... Yeo, B. T. T. (2018). Spatial topography of individual-specific cortical networks predicts human cognition, personality, and emotion. Cerebral Cortex, doi:10.1093/cercor/bhy123

Krienen, F. M., Yeo, B. T. T., \& Buckner, R. L. (2014). Reconfigurable task-dependent functional coupling modes cluster around a core functional architecture. Philosophical Transactions of the Royal Society of London. Series B, Biological Sciences, 369(1653). doi:10.1098/rstb.2013.0526

La, C., Garcia-Ramos, C., Nair, V. A., Meier, T. B., Farrar-Edwards, D., Birn, R., ... Prabhakaran, V. (2016). Age-related changes in BOLD activation pattern in phonemic fluency paradigm: An investigation of activation, functional connectivity and psychophysiological interactions. Frontiers in Aging Neuroscience, 8, 110. doi:10.3389/fnagi.2016.00110

Lancichinetti, A., \& Fortunato, S. (2012). Consensus clustering in complex networks. Scientific Reports, 2, 336.

Li, B., Wang, X., Yao, S., Hu, D., \& Friston, K. (2012). Task-dependent modulation of effective connectivity within the default mode network. Frontiers in Psychology, 3, 206. doi:10.3389/ fpsyg.2012.00206

Lohmann, G., Hoehl, S., Brauer, J., Danielmeier, C., BornkesselSchlesewsky, I., Bahlmann, J., ... Friederici, A. (2010). Setting the frame: The human brain activates a basic low-frequency network for language processing. Cerebral Cortex, 20(6), 1286-1292. doi:10.1093/cercor/bhp190

Lund, T. E., Madsen, K. H., Sidaros, K., Luo, W.-L., \& Nichols, T. E. (2006). Non-white noise in fMRI: Does modelling have an impact? Neuroimage, 29(1), 54-66.

Margulies, D. S., Böttger, J., Long, X., Lv, Y., Kelly, C., Schäfer, A., ... Villringer, A. (2010). Resting developments: A review of fMRI post-processing methodologies for spontaneous brain activity. Magnetic Resonance Materials in Physics, Biology and Medicine, 23(5-6), 289-307. doi:10.1007/s10334-0100228-5

Marques, P., Moreira, P., Magalhães, R., Costa, P., Santos, N., Zihl, J., ... Sousa, N. (2016). The functional connectome of cognitive reserve. Human Brain Mapping, 37(9), 3310-3322.

Marsolais, Y., Perlbarg, V., Benali, H., \& Joanette, Y. (2014). Agerelated changes in functional network connectivity associated with high levels of verbal fluency performance. Cortex; a Journal Devoted to the Study of the Nervous System and Behavior, 58, 123-138. doi:10.1016/j.cortex. 2014.05.007

Marstaller, L., Williams, M., Rich, A., Savage, G., \& Burianová, H. (2015). Aging and large-scale functional networks: White matter integrity, gray matter volume, and functional connectivity in the resting state. Neuroscience, 290, 369-378. doi:10. 1016/J.NEUROSCIENCE.2015.01.049

Mattar, M. G., Cole, M. W., Thompson-Schill, S. L., Bassett, D. S., \& Honey, C. J. (2015). A functional cartography of cognitive systems. PLoS Computational Biology, 11(12), doi:10.1371/ journal.pcbi.1004533

Mattis, S. (1988). Dementia rating scale: DRS: Professional manual. PAR.

Meinzer, M., Flaisch, T., Seeds, L., Harnish, S., Antonenko, D., Witte, V.,...Crosson, B. (2012). Same modulation but different starting points: Performance modulates age differences in inferior frontal cortex activity during word-retrieval. PloS One, 7(3), e33631. doi:10.1371/journal.pone.0033631

Mennes, M., Kelly, C., Colcombe, S., Castellanos, F. X., \& Milham, M. P. (2013). The extrinsic and intrinsic functional architectures of the human brain are not equivalent. Cerebral Cortex, 23(1), 223-229. doi:10.1093/cercor/bhs010

Meunier, D., Stamatakis, E. a., \& Tyler, L. K. (2014). Age-related functional reorganization, structural changes, and preserved cognition. Neurobiology of Aging, 35(1), 42-54. doi:10.1016/j. neurobiolaging.2013.07.003

Mill, R. D., Ito, T., \& Cole, M. W. (2017). From connectome to cognition: The search for mechanism in human functional brain networks. Neurolmage, 160, 124-139. doi:10.1016/j. neuroimage.2017.01.060

Monge, Z. A., \& Madden, D. J. (2016). Linking cognitive and visual perceptual decline in healthy aging: The information degradation hypothesis. Neuroscience and Biobehavioral Reviews, 69, 166-173. doi:10.1016/j.neubiorev.2016.07.031

Mowinckel, A. M., Espeseth, T., \& Westlye, L. T. (2012). Networkspecific effects of age and in-scanner subject motion: A resting-state fMRI study of 238 healthy adults. Neuroimage, 63(3), 1364-1373.

Muller, A. M., Mérillat, S., \& Jäncke, L. (2016). Older but still fluent? Insights from the intrinsically active baseline configuration of the aging brain using a data driven graphtheoretical approach. Neurolmage, 127, 346-362. doi:10. 1016/j.neuroimage.2015.12.027

Muller, A. M., \& Meyer, M. (2014). Language in the brain at rest: New insights from resting state data and graph theoretical analysis. Frontiers in Human Neuroscience, 8, 228. doi:10. 3389/fnhum.2014.00228

Murphy, K., Birn, R. M., Handwerker, D. A., Jones, T. B., \& Bandettini, P. A. (2009). The impact of global signal regression on resting state correlations: Are anti-correlated networks introduced? Neuroimage, 44(3), 893-905.

Onoda, K., Ishihara, M., \& Yamaguchi, S. (2012). Decreased functional connectivity by aging is associated with cognitive decline. Journal of Cognitive Neuroscience, 24(11), 21862198. doi:10.1162/jocn_a_00269

Park, D. C., Polk, T. A., Park, R., Minear, M., Savage, A., \& Smith, M. R. (2004). From the cover: Aging reduces neural specialization in ventral visual cortex. Proceedings of the National Academy of Sciences, 101(35), 13091-13095. doi:10.1073/ pnas.0405148101

Park, D. C., \& Reuter-Lorenz, P. (2009). The adaptive brain: Aging and neurocognitive scaffolding. Annual Review of Psychology, 60, 173-196. doi:10.1146/annurev.psych.59. 103006.093656

Perry, A., Wen, W., Kochan, N. A., Thalamuthu, A., Sachdev, P. S., \& Breakspear, M. (2017). The independent influences of age and education on functional brain networks and cognition in healthy older adults. Human Brain Mapping, 1-67. doi:10. 1002/hbm.23717

Perry, A., Wen, W., Lord, A., Thalamuthu, A., Roberts, G., Mitchell, P., ... Breakspear, M. (2015). The organisation of the elderly connectome. Neurolmage, 114, 414-426. doi:10.1016/j. neuroimage.2015.04.009

Persson, N., Ghisletta, P., Dahle, C. L., Bender, A. R., Yang, Y., Yuan, P.,... Raz, N. (2016). Regional brain shrinkage and change in cognitive performance over two years: The bidirectional influences of the brain and cognitive reserve factors. Neurolmage, 126, 15-26. doi:10.1016/j.neuroimage. 2015.11.028

Power, J. D., Barnes, K. A., Snyder, A. Z., Schlaggar, B. L., \& Petersen, S. E. (2012). Spurious but systematic correlations in functional connectivity MRI networks arise from subject 
motion. Neurolmage, 59(3), 2142-2154. doi:10.1016/j. neuroimage.2011.10.018

Power, J. D., Mitra, A., Laumann, T. O., Snyder, A. Z., Schlaggar, B. L., \& Petersen, S. E. (2014). Methods to detect, characterize, and remove motion artifact in resting state fMRI. Neurolmage, 84 , 320-341. doi:10.1016/j.neuroimage.2013.08.048

Price, C. J. (2012). A review and synthesis of the first 20 years of PET and $\mathrm{FMRI}$ studies of heard speech, spoken language and reading. Neurolmage, 62(2), 816-847. doi:10.1016/j. neuroimage.2012.04.062

Price, C. J., \& Devlin, J. T. (2003). The myth of the visual word form area. Neurolmage, 19(3), 473-481. doi:10.1016/S10538119(03)00084-3

Saad, Z. S., Gotts, S. J., Murphy, K., Chen, G., Jo, H. J., Martin, A., \& Cox, R. W. (2012). Trouble at rest: How correlation patterns and group differences become distorted after global signal regression. Brain Connectivity, 2(1), 25-32.

Sadaghiani, S., \& Kleinschmidt, A. (2013). Functional interactions between intrinsic brain activity and behavior. Neurolmage, 80, 379-386. doi:10.1016/j.neuroimage.2013.04.100

Sala-Llonch, R., Bartrés-Faz, D., \& Junqué, C. (2015). Reorganization of brain networks in aging: A review of functional connectivity studies. Frontiers in Psychology, 6, 663. doi:10.3389/fpsyg.2015.00663

Sala-Llonch, R., Smith, S. M., Woolrich, M., \& Duff, E. P. (2018). Spatial parcellations, spectral filtering, and connectivity measures in fMRI: Optimizing for discrimination. 40(2), 407419. doi:10.1002/hbm.24381

Salthouse, T. A. (1998). Independence of age-related influences on cognitive abilities across the life span. Developmental Psychology, 34(5), 851-864.

Salthouse, T. A. (2014). Quantity and structure of word knowledge across adulthood. Intelligence, 46, 122-130. doi:10. 1016/j.intell.2014.05.009

Salthouse, T. A., Hancock, H. E., Meinz, E. J., \& Hambrick, D. Z. (1996). Interrelations of age, visual acuity, and cognitive functioning. The Journals of Gerontology Series B: Psychological Sciences and Social Sciences, 51B(6), 317-333.

Salthouse, T. A., \& Kersten, A. W. (1993). Decomposing adult age differences in symbol arithmetic. Memory \& Cognition, 21(5), 699-710.

Sambataro, F., Murty, V. P., Callicott, J. H., Tan, H.-Y., Das, S., Weinberger, D. R., \& Mattay, V. S. (2010). Age-related alterations in default mode network: Impact on working memory performance. Neurobiology of Aging, 31(5), 839-852. doi:10. 1016/j.neurobiolaging.2008.05.022

Samu, D., Campbell, K. L., Tsvetanov, K. A., Shafto, M. A., CamCAN, \& Tyler, L. K. (2017). Preserved cognitive functions with age are determined by domain-dependent shifts in network responsivity. Nature Communications, 1-14. doi:10. 1038/ncomms 14743

Saur, D., Kreher, B. W., Schnell, S., Kümmerer, D., Kellmeyer, P., Vry, M.-S., ... Weiller, C. (2008). Ventral and dorsal pathways for language. Proceedings of the National Academy of Sciences of the United States of America, 105(46), 1803518040. doi:10.1073/pnas.0805234105

Schaie, K. W., \& Willis, S. L. (2010). The Seattle longitudinal study of adult cognitive development. ISSBD Bulletin, 57 (1), 24-29.

Schmitter-Edgecombe, M., Vesneski, M., \& Jones, D. W. R. (2000). Aging and word-finding: A comparison of spontaneous and constrained naming tests. Archives of Clinical
Neuropsychology, 15(6), 479-493. doi:10.1016/S0887-6177 (99)00039-6

Schultz, D. H., \& Cole, M. W. (2016). Higher intelligence is associated with less task-related brain network reconfiguration. The Journal of Neuroscience, 36(33), 8551-8561. doi:10. 1523/jneurosci.0358-16.2016

Schwartz, B. L., \& Frazier, L. D. (2005). Tip-of-the-tongue states and aging: Contrasting psycholinguistic and metacognitive perspectives. The Journal of General Psychology, 132(4), 377-391. doi:10.3200/genp.132.4.377-391

Seghier, M. L., Fagan, E., \& Price, C. J. (2010). Functional subdivisions in the left angular gyrus where the semantic system meets and diverges from the default network. The Journal of Neuroscience: The Official Journal of the Society for Neuroscience, 30(50), 16809-16817. doi:10.1523/jneurosci. 3377-10.2010

Selya, A. S., Rose, J. S., Dierker, L. C., Hedeker, D., \& Mermelstein, R. J. (2012). A practical guide to calculating Cohen's f2, a measure of local effect size, from PROC MIXED. Frontiers in Psychology, 3, 111.

Shafto, M. A., Burke, D. M., Stamatakis, E. A., Tam, P. P., \& Tyler, L. K. (2007). On the tip-of-the-tongue: Neural correlates of increased word-finding failures in normal aging. Journal of Cognitive Neuroscience, 19(12), 2060-2070. doi:10.1162/jocn. 2007.19.12.2060

Shafto, M. A., James, L. E., Abrams, L., \& Tyler, L. K. (2016). Agerelated increases in verbal knowledge are not associated with word finding problems in the Cam-CAN cohort: What you know won't hurt you. The Journals of Gerontology Series B: Psychological Sciences and Social Sciences, 72(1), 100-106. doi:10.1093/geronb/gbw074

Shafto, M. A., \& Tyler, L. K. (2014). Language in the aging brain: The network dynamics of cognitive decline and preservation. Science, 346(6209), 583-587.

Shah, L. M., Cramer, J. A., Ferguson, M. A., Birn, R. M., \& Anderson, J. S. (2016). Reliability and reproducibility of individual differences in functional connectivity acquired during task and resting state. Brain and Behavior, 456, 1-15. doi:10.1002/brb3.456

Shine, J. M., Bissett, P. G., Bell, P. T., Gorgolewski, K. J., Moodie, C. A., Poldrack, R. A., ... Balsters, J. H. (2016). Supplementary info: The dynamics of functional brain networks: Integrated network states during cognitive task article the dynamics of functional brain networks: Integrated network states during cognitive task performance. Neuron, 92(2), 544-554. doi:10.1016/j.neuron.2016.09.018

Siedlecki, K. L., Stern, Y., Reuben, A., Sacco, R. L., Elkind, M. S. V., \& Wright, C. B. (2009). Construct validity of cognitive reserve in a multiethnic cohort: The Northern Manhattan study. Journal of the International Neuropsychological Society, 15(04), 558-569.

Sporns, O. (2014). Contributions and challenges for network models in cognitive neuroscience. Nature Neuroscience, 17 (5), 652-660. doi:10.1038/nn.3690

Sporns, O., \& Betzel, R. F. (2016). Modular brain networks. Annual Review of Psychology, 67(1), 613-640. doi:10.1146/ annurev-psych-122414-033634

Spreng, R. N., \& Grady, C. L. (2010). Patterns of brain activity supporting autobiographical memory, prospection, and theory of mind, and their relationship to the default mode network. Journal of Cognitive Neuroscience, 22(6), 11121123. doi:10.1162/jocn.2009.21282 
Spreng, R. N., \& Schacter, D. L. (2012). Default network modulation and large-scale network interactivity in healthy young and old adults. Cerebral Cortex, 22(11), 2610-2621. doi:10.1093/cercor/bhr339

Stanley, M. L., Dagenbach, D., Lyday, R. G., Burdette, J. H., \& Laurienti, P. J. (2014). Changes in global and regional modularity associated with increasing working memory load. Frontiers in Human Neuroscience, 8, 954.

Steffener, J., Barulli, D., Habeck, C., O'Shea, D., Razlighi, Q., Stern, Y., ... Bosch, B. (2014). The role of education and verbal abilities in altering the effect of age-related gray matter differences on cognition. PLoS ONE, 9(3), e91196. doi:10.1371/ journal.pone.0091196

Steffener, J., Reuben, A., Rakitin, B. C., \& Stern, Y. (2011). Supporting performance in the face of age-related neural changes: Testing mechanistic roles of cognitive reserve. Brain Imaging and Behavior, 5(3), 212-221.

Steffener, J., \& Stern, Y. (2012). Exploring the neural basis of cognitive reserve in aging. Biochimica et Biophysica Acta (BBA) Molecular Basis of Disease, 1822(3), 467-473. doi:10.1016/j. bbadis.2011.09.012

Stern, Y. (2009). Cognitive reserve. Neuropsychologia, 47(10), 2015-2018. doi:10.1016/j.neuropsychologia.2009.03.004

Stern, Y., Gazes, Y., Razlighi, Q., Steffener, J., \& Habeck, C. (2018). A task-invariant cognitive reserve network. Neurolmage, 178, 36-45. doi:10.1016/j.neuroimage.2018.05.033

Stern, Y., Habeck, C., Steffener, J., Barulli, D., Gazes, Y., Razlighi, Q.,... Salthouse, T. (2014). The reference ability neural network study: Motivation, design, and initial feasibility analyses. Neurolmage, 103, 139-151. doi:10.1016/j.neuroimage. 2014.09.029

Taylor, J. R., Williams, N., Cusack, R., Auer, T., Shafto, M. A., Dixon, M., ... Henson, R. N. (2015). The Cambridge Centre for ageing and neuroscience (Cam-CAN) data repository: Structural and functional MRI, MEG, and cognitive data from a cross-sectional adult lifespan sample. Neurolmage, 5-12. doi:10. 1016/j.neuroimage.2015.09.018

Tohka, J., Zijdenbos, A., \& Evans, A. (2004). Fast and robust parameter estimation for statistical partial volume models in brain MRI. Neurolmage, 23(1), 84-97. doi:10.1016/j. neuroimage.2004.05.007

Tomasi, D., \& Volkow, N. D. (2010). Functional connectivity density mapping. Proceedings of the National Academy of Sciences, 107(21), 9885-9890. doi:10.1073/pnas.1001414107

Tomasi, D., \& Volkow, N. D. (2012a). Aging and functional brain networks. Molecular Psychiatry, 17(5), 471, 549-58. doi:10. 1038/mp.2011.81

Tomasi, D., \& Volkow, N. D. (2012b). Language network: Segregation, laterality and connectivity. Molecular Psychiatry, 17(8), 759. doi:10.1038/mp.2012.99

Tucker-Drob, E. M., \& Salthouse, T. A. (2011). Individual differences in cognitive aging. In The Wiley-Blackwell handbook of individual differences (pp. 242-267). Hoboken, NJ: WileyBlackwell.

Turken, A. U., \& Dronkers, N. F. (2011). The neural architecture of the language comprehension network: Converging evidence from lesion and connectivity analyses. Frontiers in Systems Neuroscience, 5(1). doi:10.3389/fnsys.2011.00001

Tyler, L. K., Wright, P., Randall, B., Marslen-Wilson, W. D., Stamatakis, E. A., Ashburner, J.,... Haupt, W. (2010). Reorganization of syntactic processing following left-hemisphere brain damage: Does right-hemisphere activity preserve function? Brain: A Journal of Neurology, 133(11), 3396-3408. doi:10.1093/brain/awq262

United Nations. (2017). World population ageing. Retrieved from http://www.un.org/en/development/desa/population/ publications/pdf/ageing/WPA2017_Highlights.pdf

Urchs, S., Armoza, J., Benhajali, Y., St-Aubin, J., Orban, P., \& Bellec, P. (2017). MIST: A multi-resolution parcellation of functional brain networks. MNI Open Research, 1, 3.

Verhaegen, C., \& Poncelet, M. (2013). Changes in naming and semantic abilities with aging from 50 to 90 years. Journal of the International Neuropsychological Society: JINS, 19(2), 119-126. doi:10.1017/S1355617712001178

Verhaeghen, P. (2003). Aging and vocabulary scores: A metaanalysis. Psychology and Aging, 18, 332-339.

Wang, J., Han, J., Nguyen, V. T., Guo, L., \& Guo, C. C. (2017). Improving the test-retest reliability of resting state $\mathrm{fMRI}$ by removing the impact of Sleep. Frontiers in Neuroscience, 11, 249. doi:10.3389/fnins.2017.00249

Wechsler, D. (1997). WAIS-3., WMS-3: Wechsler adult intelligence scale, Wechsler memory scale: Technical manual, P. Corporation (Ed.).

WHO | What is Healthy Ageing? (2018). Retrieved from http:// www.who.int/ageing/healthy-ageing/en/

Wierenga, C. E., Benjamin, M., Gopinath, K., Perlstein, W. M., Leonard, C. M., Rothi, L. J. G., ... Crosson, B. (2008). Agerelated changes in word retrieval: Role of bilateral frontal and subcortical networks. Neurobiology of Aging, 29(3), 436-451. doi:10.1016/j.neurobiolaging.2006.10.024

Wirth, M., Jann, K., Dierks, T., Federspiel, A., Wiest, R., \& Horn, H. (2011). Semantic memory involvement in the default mode network: A functional neuroimaging study using independent component analysis. Neurolmage, 54(4), 3057-3066. doi:10.1016/J.NEUROIMAGE.2010.10.039

Woodcock, R. W., Johnson, M. B., \& Mather, N. (1989). WoodcockJohnson psycho-educational battery-revised: WoodcockJohnson tests of cognitive ability. Allen, TX: DLM Teaching Resources.

Xiang, H., Dediu, D., Roberts, L., Norris, D. G., \& Hagoort, P. (2012). The structural connectivity underpinning language aptitude, working memory, and IQ in the Perisylvian language network. Language Learning, 62(Suppl.2), 110-130.

Xiang, H.-D., Fonteijn, H. M., Norris, D. G., \& Hagoort, P. (2010). Topographical functional connectivity pattern in the perisylvian language networks. Cerebral Cortex, 20(3), 549-560. doi:10.1093/cercor/bhp119

Yan, C.-G., Cheung, B., Kelly, C., Colcombe, S., Craddock, R. C., Di Martino, A., ... Milham, M. P. (2013). A comprehensive assessment of regional variation in the impact of head micromovements on functional connectomics. Neurolmage, 76, 183201. doi:10.1016/j.neuroimage.2013.03.004 